\title{
Efficient Mechanical Stress Transfer in Multilayer Graphene with a Ladder-like Architecture
}

\author{
Aristotelis P. Sgouros, Charalampos Androulidakis, Georgia Tsoukleri, George \\ Kalosakas, Nikos Delikoukos, Stefano Signetti, Nicola M. Pugno, John Parthenios, \\ Costas Galiotis, and Konstantinos Papagelis*
}

Cite This: ACS Appl. Mater. Interfaces 2021, 13, 4473-4484 Read Online

\begin{tabular}{l|l|llll}
\hline ACCESS $\mid$ 进 & Metrics \& More & Article Recommendations & & \\
\hline
\end{tabular}

ABSTRACT: We report that few graphene flakes embedded into polymer matrices can be mechanically $\overline{L_{0}} \overline{\text { stretched to }}$ relatively large deformation $(>1 \%)$ in an efficient way by adopting a particular ladder-like momphotogy consisting of consecutive mono-, bi-, tri-, and four-layer graphene units. In this type of flake architecture, all the surrounding polymer inducing similar deformation on the individual graphene layers, preventimg intertayer stiding and optimizing the strain transfer efficiency. We have exploited Raman spectroscopy to quantify this-effect from a mechanical standpoint. The finite element method and molecular dynamics simulations have been used to interpiret the above experimental findings. The results suggest that a step pyramid-like architecture of a flake can be ideal foreffieient loading of layered materials embedded into a polymer and that there are two prevailing mechanisms that govermaxiatstress tramsfer, namely, interfacial shear transfer and axial transmission through the ends. This concept can be dimensional materials and related van der Waals heterostructures fabricated either by mechanicatexfotiation or chemrical vapor deposition by appropriate patterning. This work opens new perspectives in numerous applications, (including high volume fraction composites, flexible electronics, and straintronic devices. KEYWORDS: multilayer graphene, Raman spectroscopy, mechanical strain, load transfer, nanocomposites modeling

Graphene reinforced polymer composites show high potential demonstrated that an optimal number of layers ( 5-10) for commercialization, and several products such as sports and maximizes the specific energy dissipation by FLG armors ${ }^{8}$ recreational goods (bicycle tires, tennis rackets, etc.) have subjected to hypervelocity impact load.

already been developed to satisfy consumer needs with Interestingly, 1LG embedded in a polymer matrix becomes products of superior performance. ${ }^{1}$ However, there are still significantly perturbed due to the processing conditions, the ${ }^{-11}$ several important issues to tackle before graphene is trans- emergence of defects, ${ }^{9}$ the diversity of interfacial formed into an engineering material suitable for the realization interactions, ${ }^{12}$ and the low bending rigidity of the material,

of nanocomposites on a mass-scale production.

The preparation strategies for the production of few-layer graphene (FLG) or thicker nanographite platelets significantly outbalance those of monolayer flakes, ensuring reasonable cost, bulk quantities, and scalability. Bilayer (2LG), trilayer (3LG), four-layer (4LG), and multilayer graphene (MLG) up to about 10 layers exhibits excellent electronic, optical, and vibrational properties distinct from those of single-layer graphene (1LG), which strongly depend on the crystallographic and twisted stacking of the individual layers, providing an additional degree

of freedom to tune multifunctional properties. ${ }^{2}$ However, their overall mechanical properties are hampered by poor mechanical transfer and interlayer sliding. ${ }^{3-5}$ Moreover, twisted graphene bilayers can become superconductive by twisting them at a "magic angle" equal to $1.1^{\circ},{ }^{6}$ whilst Yankowitz et al. ${ }^{7}$ demonstrated that varying the interlayer coupling through the application of hydrostatic pressure allows which in turn deteriorate its superior properties. On the other hand, the incorporation of graphene as a transparent conductive film in flexible optoelectronic devices, e.g., polymer-based touch panel displays, generally favored fewlayer chemical vapor deposition (CVD) sheets since, among other properties, the sheet resistivity scales inversely proportional to the number of layers $\left(\mathrm{N}_{\mathrm{L}}\right) \cdot{ }^{13}$ Therefore, in real applications, the implementation of MLG seems to be a feasible and promising choice and it is of paramount 


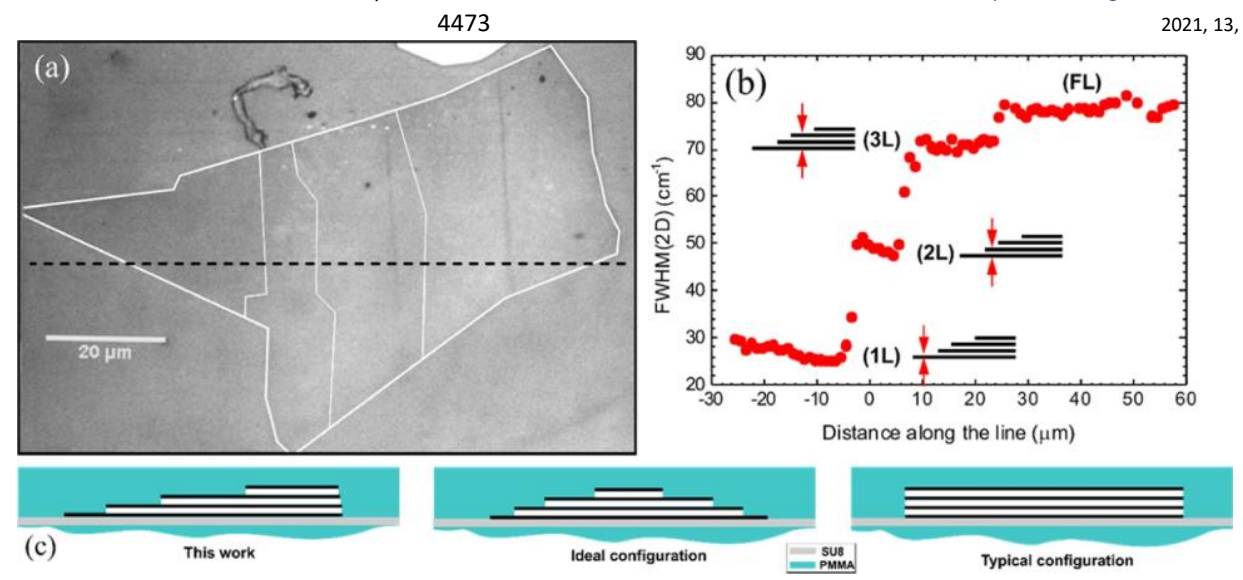

Figure 1. (a) Optical micrograph of the embedded graphene flake with dimensions of about $100 \times 50 \mu \mathrm{m}^{2}$. (b) FWHM of the $2 \mathrm{D}$ peak along the black dashed line shown in (a). (c) Schematic illustrations of the ladder-like flake examined in the present work (left), ideal flake with a pyramidlike architecture where the graphene layers are stacked symmetrically (center) and a typical multilayer configuration (right).

importance to tailor the ability of MLG flakes to optimally reinforce a polymer and guarantee efficient multifunctional composites at the same time.

The performance of graphene as a reinforcement agent has been intensively studied, simulating those conditions that can be found in the interior of bulk nanocomposites, obtained via fabrication of model composites, for example, by depositing short flakes of graphene or continuous CVD material on a polymeric substrate and cover it with polymeric layer(s) using spin coating. Raman spectroscopy and especially its variant strain (uniaxial or biaxial) mapping and strain-induced phonon mode sensitivities have been successfully employed to evaluate the reinforcement efficiency of various graphene types in a range of polymer matrices for strain up to the order of $1 \% \cdot{ }^{14-28} \mathrm{~A}$ key finding in these studies is that the graphene- polymer interactions are strong for 1LG and 2LG, while the stress transfer between the individual layers in FLG is poor. In a polymer composite filled with FLGs, the applied stress is transmitted from the matrix through shear forces to the outer layers and, in turn, transferred to the inner layers again by shear through the weak van der Waals (vdW) interactions that hold the graphene layers together. In a $3 \mathrm{LG}$ under strain, the atomic registry of the graphene layers is distorted even at low strain. ${ }^{29}$ This is the reason why graphite and other layered materials such as molybdenum disulfide can be used as agents in solid lubricants. ${ }^{30}$ For a fully embedded bilayer, both outer layers are adhered to the polymer and, thus, the bilayer is

$\mathrm{su}^{\mathrm{ffi}}$ ciently deformed. ${ }^{29,31}$

This work aims to propose a specific design of few-layer graphene (FLG) structures, which when embedded into polymer matrices can be uniformly stretched up to large deformation, overcoming the inherent weak interlayer stresstransfer efficiency, thus maximizing the strain energy per unit volume in the flake. Very recently, we have shown ${ }^{28}$ by means of Raman spectroscopy that thermally induced wrinkles in exfoliated 3LG flakes enhance the load-bearing capacity of FLGs as compared to previous results using flat flakes. This performance was attributed to the significant increase of the graphene/polymer interfacial shear stress due to small wavelength corrugations. The current work represents a significant leap forward regarding the efficient use of the multilayer material in numerous applications such as the usage of thicker flakes to achieve a similar level of mechanical performance of the monolayer but with richer functionalities, the production of polymer composites with higher volume fractions and better mechanical response, and the fabrication of "straintronic" devices based on suitable engineering of mechanical deformations. The proposed methodology can also be applied to other two-dimensional (2D) materials as well as their heterostructures produced either by mechanical exfoliation or CVD. Therefore, overcoming the poor mechanical performance due to the weak coupling between the adjacent layers will enhance the mechanical stability of the heterostructure assemblies, and hence their optoelectronic performance, enriching the functionalities of the $\mathrm{vdW}$

$32-35$ heterostructure-based devices.

\section{RESULTS AND DISCUSSION}

The fabrication strategy of monolayer and multilayer 2D materials by mechanical exfoliation (top-down approach) is essential to understand their fundamental properties, to improve the metrology for applications and quality control. ${ }^{2}$ The ultrathin nature of 2D materials creates obstacles in the path to consider exfoliated crystals as a model system for 2D materials research due to the sensitivity of graphene to the surrounding environment, doping (substrate induced or chemical), ${ }^{36}$ and the transferring 
procedure after exfoliation. ${ }^{37,38}$ Therefore, comparing measurements from different samples is questionable even if they possess the same architecture. In the past, ${ }^{29,31}$ we have addressed the problem of differences among various literature works in the uniaxial strain rates of the main Raman peaks in $2 \mathrm{LG}$ and $3 \mathrm{LG}$ graphene. We did not study isolated 2LG and 3LG flakes but flakes of a particular shape architecture, where the bilayer and trilayer domains covered only a fraction of a larger monolayer. In this way, we eliminated the sample effect, enabling the direct comparison between the strain response of flakes of various thicknesses. In the current work, we have moved a step forward investigating a unique sample architecture of an elongated, large area ladder-like flake, as shown in the optical microscopy image in Figure 1a. It is a multilayer flake with dimensions of about $100 \mu \mathrm{m}$ in length and $50 \mu \mathrm{m}$ in width, which has been fully embedded into a poly(methyl methacrylate) (PMMA) matrix (see Methods section). Raman analysis based on the full width at half-maximum (FWHM) of the 2D peak (see Figure 1b) shows a significant variation across the marked line depicted in Figure 1a. The flake consists of 1LG, 2LG, 3LG, and 4LG, consecutively, in a ladder-like manner. Further
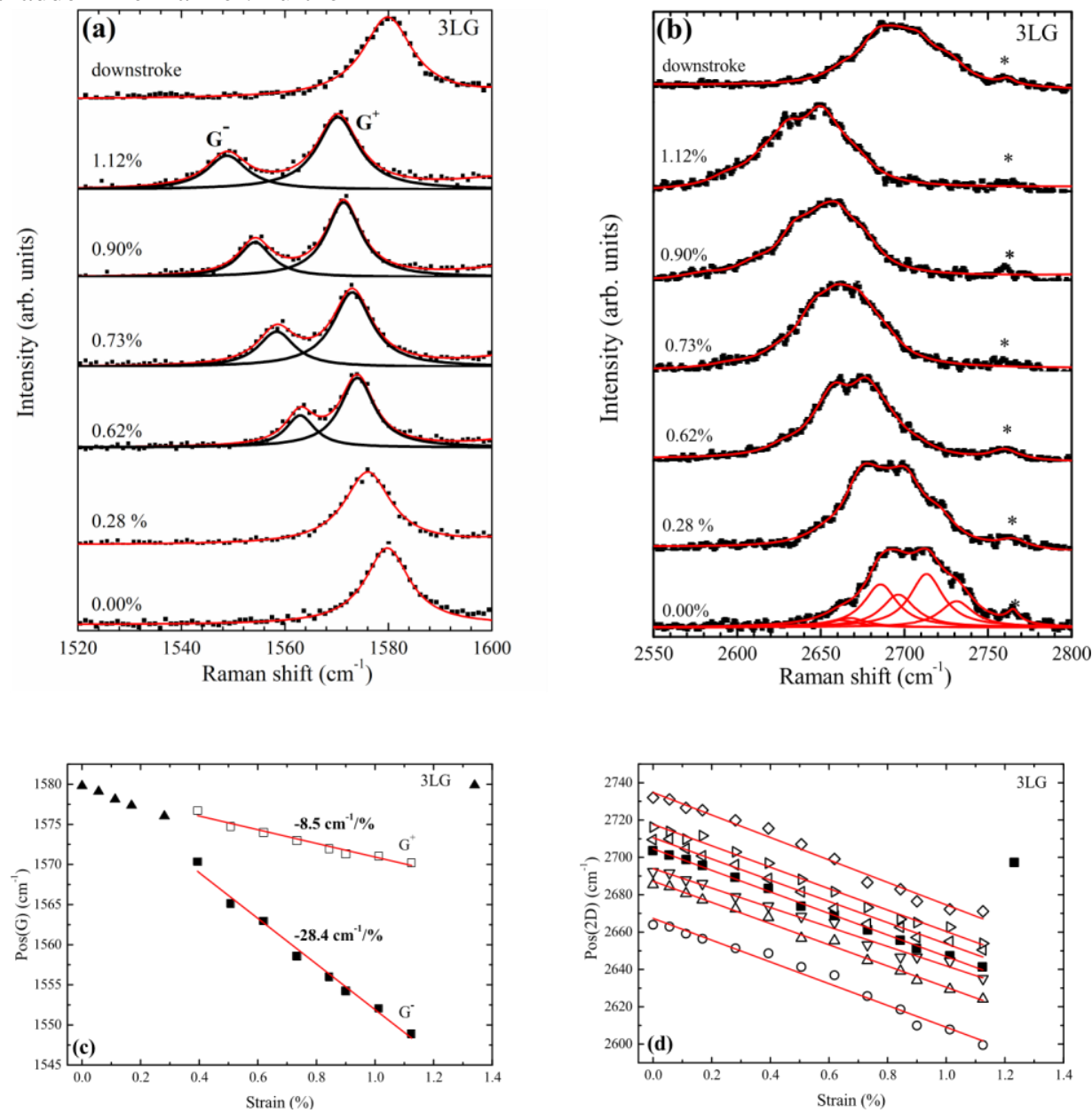

Figure 2. Raman spectra of the trilayer part of the ladder-like sample excited at $514.5 \mathrm{~nm}(2.41 \mathrm{eV})$ recorded upon uniaxial tensile strain increase for (a) the $\mathrm{G}$ and (c) the 2D peak regions. The top spectrum in both cases recorded after strain release. The asterisk $\left({ }^{*}\right)$ in $(\mathrm{c})$ denotes a Raman peak at $2765 \mathrm{~cm}^{-1}$ originated from the PMMA covering layer. (b) G-band Raman frequencies at various strain levels of the trilayer part of the ladder-like sample at room temperature. An onset of splitting is observed at $0.3 \%$. (d) Uniaxial strain dependence of the six Lorentzian components (open symbols) that constitute the Raman 2D peak for the 3LG. The solid symbols correspond to the fitting of the 2D peak by a single Lorentzian function.

details on the Raman mapping and optical contrast difference between the graphene layers and the substrate that clearly identifies the number of layers, shape, and size of the corresponding graphene domains are presented in the Supporting Information (Figures S1 and S2). 
Table 1. Strain Slopes of $G^{ \pm}$and $2 D^{ \pm}$Raman Peaks and the Maximum Applied Strain with Respect to the Excitation Wavelength, the Number of Layers, and the Presence or Absence of the Cover Layer

\begin{tabular}{|c|c|c|c|c|c|c|c|}
\hline \multirow[b]{3}{*}{ excitation wavelength $(\mathrm{nm})$} & \multirow[b]{3}{*}{ no. of layers $\mathrm{N}_{\mathrm{L}}$} & \multirow[b]{3}{*}{ cover layer } & \multicolumn{3}{|c|}{ slope $\left(\mathrm{cm}^{-1} / \%\right)$} & \multirow[b]{3}{*}{$2 \mathrm{D}^{+}$} & \multirow[b]{3}{*}{$\begin{array}{l}\max \text { strain } \\
(\%)\end{array}$} \\
\hline & & & \multirow[b]{2}{*}{ G- } & \multicolumn{2}{|r|}{$2 \mathrm{D}$} & & \\
\hline & & & & $\mathrm{G}_{+}$ & $2 \mathrm{D}^{-}$ & & \\
\hline 514.5 & 141 & no & -31.7 & -10.8 & -64 & & 1.3 \\
\hline 514.5 & 114 & yes & & & 65.9 & & 1.3 \\
\hline 532 & 139 & no & -14.2 & & -27.8 & & 0.8 \\
\hline 532 & 142 & no & -12.5 & -5.6 & -21 & & 1.3 \\
\hline 785 & 140 & no & & & -48.8 & & 0.4 \\
\hline 785 & 140 & yes & & & -57.7 & & 0.4 \\
\hline
\end{tabular}




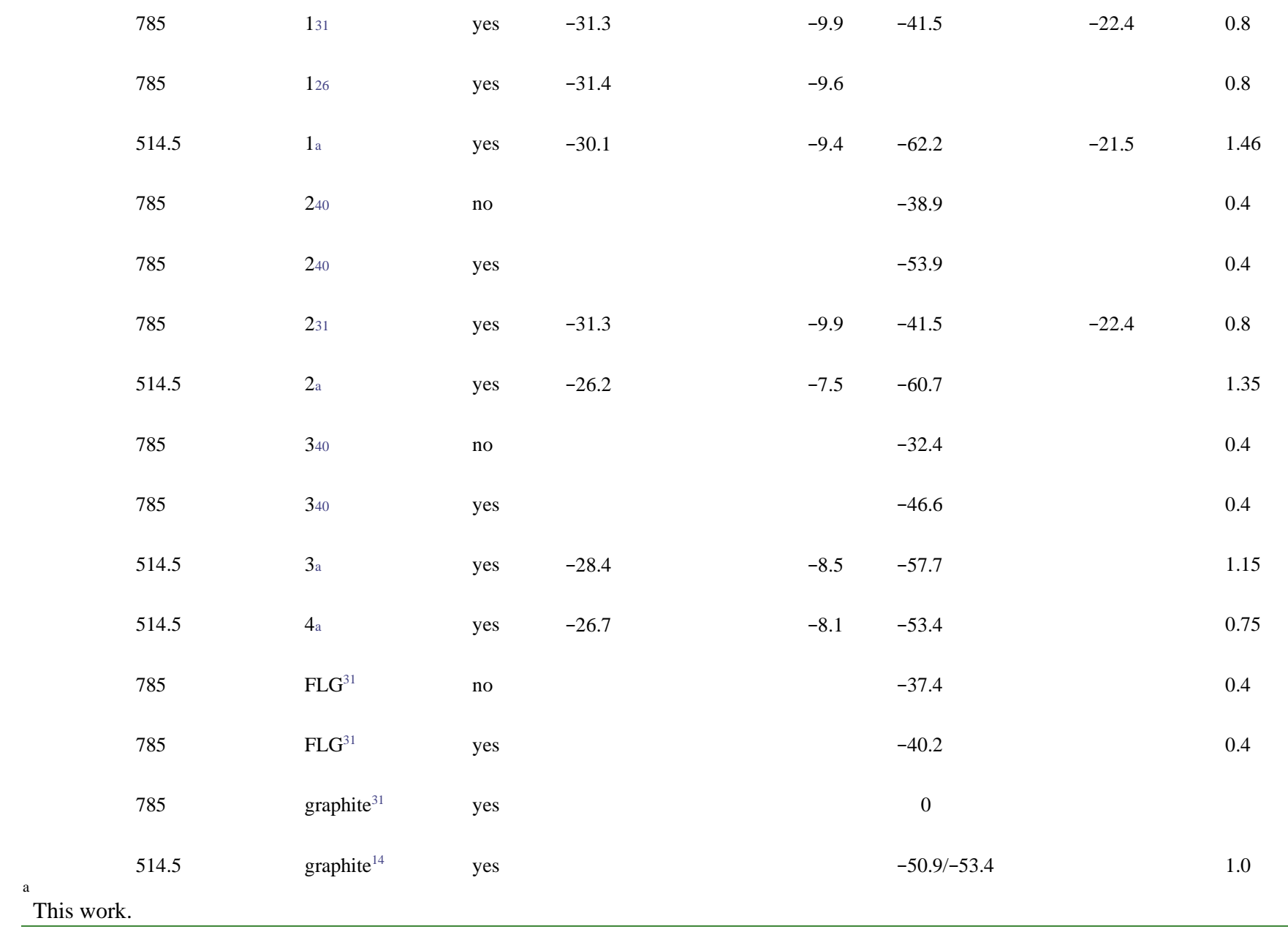

The strain/stress transfer efficiency was assessed through the slope of both G and 2D peak shifts with applied strain. In the Supporting Information (Sections S2-S6), a detailed analysis of the strain-induced response of the G and 2D Raman peaks of 1LG, 2LG, 3LG, and 4LG parts of the ladder-like sample is presented. Here, we discuss some key points regarding mainly the G-peak for which the interpretation of Raman response as a function of tensile strain is more straightforward. A lifting of the degeneracy of the G-peak for all 1L-4LG domains is observed (Figures 2, S3, S4, and S6 in the Supporting Information Sections S2-S4 and S6) upon uniaxial loading. The mean strain sensitivities of the G-peak in all cases are similar to the corresponding values for the 1LG and 2LG (see Table 1). In Figure 2, we present data for the mechanical response in 3LG (see discussion in the Supporting Information Section S4). It is important to underline the higher strain sensitivity here $\left(-8.5\right.$ and $-28.4 \mathrm{~cm}^{-1} / \%$ for the $\mathrm{G}^{+}$and the $\mathrm{G}^{-}$components and $-57.7 \mathrm{~cm}^{-1} / \%$ for the $2 \mathrm{D}$ band) compared with previously reported values ${ }^{24,29,39}$ for $3 \mathrm{LG}$ under uniaxial tension. In addition, the G-band splitting for 4LG (Figure S6a in the Supporting Information) is presented for the first time in the literature, indicating ideal stress transfer from the matrix to the whole graphene block. The maximum applied strain in $1 \mathrm{LG}$ and $2 \mathrm{LG}$ is $\sim 1.46$ and $\sim 1.35 \%$, while in $3 \mathrm{LG}$ and $4 \mathrm{LG}$ is $\sim 1.15$ and $\sim 0.75 \%$, respectively (Table 1 ). To the best of our knowledge, these are the highest reported values for uniaxial loading of multilayer graphene. For the case of $4 \mathrm{LG}$, such values are reported for the first time. The maximum applied strain is considered as the value where an abrupt upshift of the Gpeak occurs, indicating the failure of the flake/polymer interface (Figure 2, and Supporting Information Figures S3, S4, and S6). Moreover, loss of Bernal stacking is observed for $3 \mathrm{LG}$ at $\sim 0.73 \%$ (Figure $2 \mathrm{~b}$ ), a value which is almost twice compared to the one reported by Gong et al., ${ }^{23}$ indicating the structural robustness of the ladder-like architecture considered here. It should be stressed that straininduced lifting of the degeneracy of the G-band in two components $\left(\mathrm{G}^{+}\right.$and $\left.\mathrm{G}^{-}\right)$is crucial for the fundamental distinction of the type of strain (biaxial or uniaxial) applied on a particular graphene sample. This behavior, along with the linear dependence of the $\mathrm{G}^{ \pm}$evolution as well as the magnitude of the corresponding strain coefficients (see Supporting Information Sections S2-S4 and S6 for details), indicates the efficiency of stress transfer in the studied ladder geometry. In addition, the large values of Raman shifts confirm that the flake can fully support the transmitted mechanical load and can be used to assess the quality of the graphene/polymer interface and the strain transfer efficiency in the model polymer composites (vide infra).

A survey of the literature for the $\mathrm{G}$ and 2D mode strain sensitivities of 1LG, 2LG, 3LG, and MLG graphene samples, including those found in this work, albeit for different Raman excitation lines, is presented in Table 1. A careful inspection of 
the quoted values reveals that the strain sensitivities of the main peaks (G, 2D) of graphene in earlier publications differ by a factor of 2 or even more. In addition, the coating (embedment) of the flake plays a major role due to the stress transfer mechanism, minimizing the possibility of slippage during loading. In contrast, in this work, the shift rates of the $\mathrm{G}^{+}$and $\mathrm{G}^{-}$ components and the 2D peak for 1LG-3LG are quite similar, being the maximum achievable in the literature. However, for the $4 \mathrm{LG}$, the shift rate of the $2 \mathrm{D}$ peak of the ladder-like sample is slightly lower, indicating, possibly, the existence of an upper limit of stress transfer efficiency as a function of the number of layers $\left(\mathrm{N}_{\mathrm{L}} \geq 4\right)$.

From Table 1, it is evident that the shift rate of the 2D peak tends to decrease by increasing the number of layers up to a strain of about $1.5 \%$. For few-layer graphene embedded into the polymer matrix, the stress is transferred from the matrix to the outer graphene layer, and this, in turn, is transferred to the inner layers by shear. ${ }^{40}$ The relatively low value $(\sim 5 \mathrm{GPa})$ of the shear modulus of the layer-to-layer interface (i.e., parallel to the layers, $\mathrm{C}_{44}$ ), governs the efficiency of the stress transfer upon the number of layers in a uniaxially strained embedded graphene. Kinloch et al. ${ }^{40}$ employed a phenomenological model similar to that proposed earlier for multiwall carbon nanotubes (CNTs), which explains the loss of axial stiffness as the number of layers increase $\left(\mathrm{N}_{\mathrm{L}}>2\right)$ as a result of the inefficient stress transfer from the outer to the inner layers.

As mentioned above, for the ladder-type structure examined in this work, the strain sensitivities of both $G$ and 2D peaks of $3 \mathrm{LG}$ and $4 \mathrm{LG}$ are similar to those measured for $1 \mathrm{LG}$. The effective mechanical loading of the $1-4 \mathrm{LG}$ is attributed to the particular morphology of the whole graphene unit (Figure 1c, left). The stress is not only transmitted to the middle layers from the outer ones through the weak vdW forces, but as is evident, part of every individual layer is in contact with the polymer and, hence, it is also stretched by interfacial shear. Therefore, this type of flake architecture induces similar deformation on the individual graphene layers, preventing interlayer sliding and thus optimizing the strain transfer efficiency in model graphenebased nanocomposites. The absence of sliding between the layers in the ladder structure gives new perspectives in the design of graphene-based nanocomposites and flexible electronics.

Taking into account the variability and randomness in graphite crystal mechanical exfoliation, the deterministic production of the ladder-like flakes is a cumbersome process. However, in the Supporting Information (Section S5), we present an additional ladder-like sample consisting of a $1 \mathrm{~L}-3 \mathrm{~L}$ and a multilayer part subjected to uniaxial compression (Figure S5a). It is noteworthy that Hooke's law (linear elasticity) applies at low strain and the response in tension and compression is symmetric in the vicinity of zero strain. We measured the 2D band sensitivity (Figure S5b) under compression of the 3LG and, as expected, the compressive strain causes a linear phonon hardening at a rate of -59.5

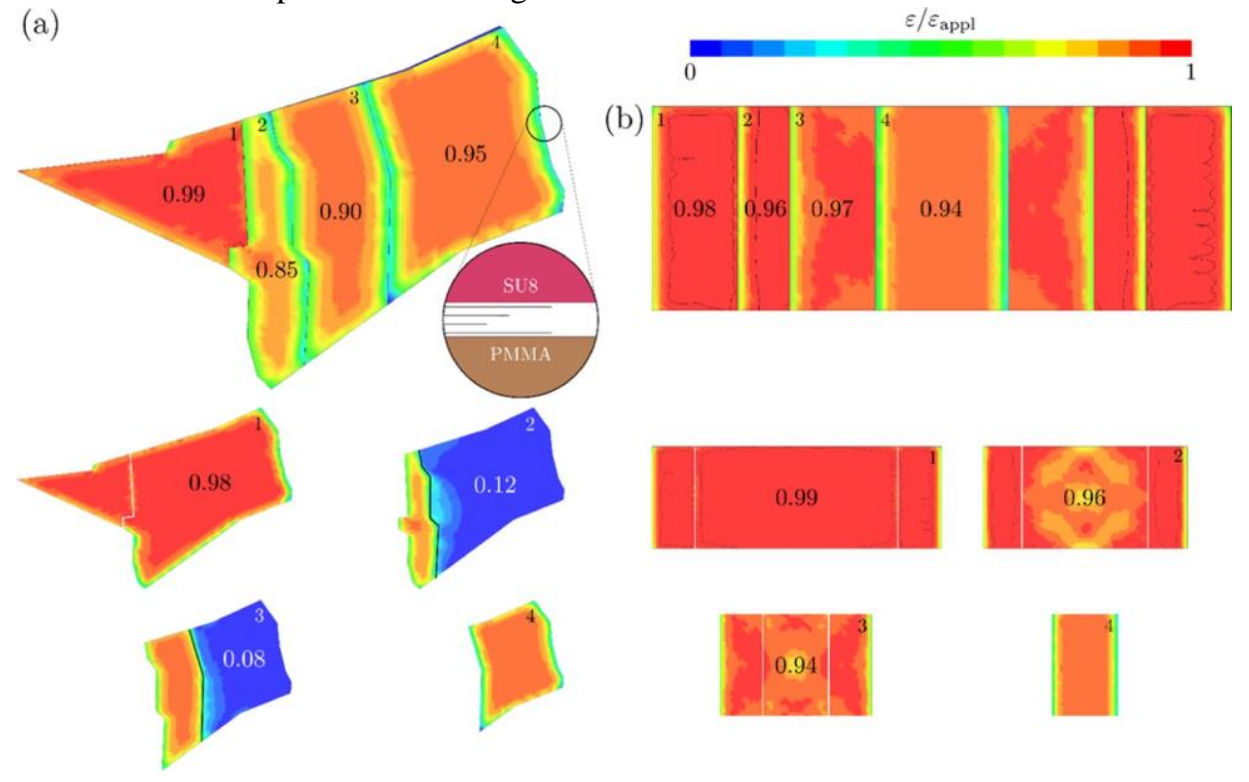

Figure 3. Results of FEM simulations. (a) Contour plot of equivalent strain $\varepsilon$ (von-Mises) within the experimental flake embedded in the SU8PMMA matrix. A magnification of the region of the real flake on the nonladdered side is reported, showing the slippage of the two innermost layers of graphene. (b) Double-ladder ideal flake. The load is applied along the horizontal direction (see Figure S7 in the Supporting Information). On each layer, we also indicate the ratio between the average (spatial) strain within the flake and the applied strain $\left(\langle\varepsilon\rangle / \varepsilon_{\text {appl }}\right)$. In the top panel, the values refer to the visible areas in contact with the matrix, while in the bottom one, the values refer to the nonvisible regions, which for layers 2 and 3 corresponds to the area not in contact with the matrix.

$\mathrm{cm}^{-1} / \%$ (Figure S5c) up to about $-0.15 \%$, where a buckling instability occurs. ${ }^{14,29,31,43}$ Therefore, the observed high phonon sensitivity under compression, similar to that of an individual $1 \mathrm{LG},{ }^{14,29,31,43}$ further justi ${ }^{\mathrm{fi}}$ es the $\mathrm{e} \mathrm{ffi}_{\text {cient }}$ stress transfer in this particular flake architecture. 
It has been proved ${ }^{40,42}$ that the axial stress is transferred to an embedded single graphene flake through shear at the graphene-polymer interface. Similar to fibrous composites, to efficiently transfer the applied strain at the composite in graphene, a critical length of reinforcement $\left(\mathrm{L}_{c}\right)$ ]defined as twice the distance from the graphene edge toward the inner part of the flake until the strain reaches its maximum value of at least $10 \mu \mathrm{m}$ is usually needed. ${ }^{44}$ As a consequence, if the available length of a graphene flake for stress transfer in the axial direction is less than $\mathrm{L}_{\mathrm{c}} / 2$ for an applied stress/strain at the composite, then only a fraction of the applied stress/strain is transmitted to the flake, decreasing the measured Raman mode strain sensitivities accordingly. However, in multilayer graphene flakes with the structure (nonladder) as shown in the right panel of Figure 1c, the shear mechanism is not capable of transmitting strain to the whole unit since the inner layers have no direct interface with the polymer. This is one of the main reasons for the relatively low and scattered strain sensitivity values of the main Raman peaks summarized in Table 1. As far as the shear mechanism is concerned, such as occurs in the ladder-like configurations, the overall length of the graphene- polymer interface along the load application direction (Figure 1a) is higher than $10 \mu \mathrm{m}$ for all layers in our test cases, allowing efficient stress/strain transfer in the exposed part. Therefore, it is important to address the transfer mechanisms that are responsible for the relatively high strain levels measured in the nonexposed parts of the ladderlike flakes. ${ }^{29}$ The pure shear mechanism cannot be involved, as shown later by finite element simulations. It is worth mentioning here that in the past we have observed ${ }^{14}$ a dramatic improvement in the stress transfer for bulk graphite embedded into a thin layer of polymer (Table 1) under tensile strain using the 2D band as a strain gauge. Also, it has been recently reported by Androulidakis et al. ${ }^{44}$ that normal stress could be transmitted directly to the embedded graphene depending on specimen fabrication and handling. To assess the contribution of axial stress generated by shear loading vis-a- vis those transmitted directly to the embedded graphene through adhesion at the ends in our ladder-like architecture, we have analyzed the problem at two different length scales and approaches. We have developed a finite element method (FEM) model that assumes pure shear transfer and molecular dynamics (MD) simulations accounting also for direct stress transmission through the ends.

Finite Element Analysis. By performing FEM simulations, we investigated the role of the geometrical conformation of the flake on its efficiency in transferring the load by the shear mechanism. Details of modeling the dispersion forces are presented in the Methods section. First, we modeled the exact geometry of the experimentally investigated flake acquired from the optical microscopy image of Figure 1a (see Figure S7 in the Supporting Information). In Figure 3a, the strain field is depicted, normalized with respect to the strain applied at the boundary, $\varepsilon_{\text {appl. }}$. It is evident that the weak vdW interaction between the graphene layers does not allow the complete load transfer also in the nonexposed part of the innermost layers ( 2 and 3 in Figure $3 \mathrm{a}$ ), which are bound to the matrix at one of their sides for a limited area. Only the relatively large area of uppermost and lowermost layers (Figure 3a), which are in direct contact with the matrix (strong bond), is able to carry a significant amount of the applied load. Layers 2 and 3 display a relatively short transfer length $(\sim 8 \mu \mathrm{m}$ and $17 \mu \mathrm{m}$, respectively), but sufficient to almost reach the applied strain locally at the center of the exposed part. This result is also obtained by a similar configuration with a single-side ladder-
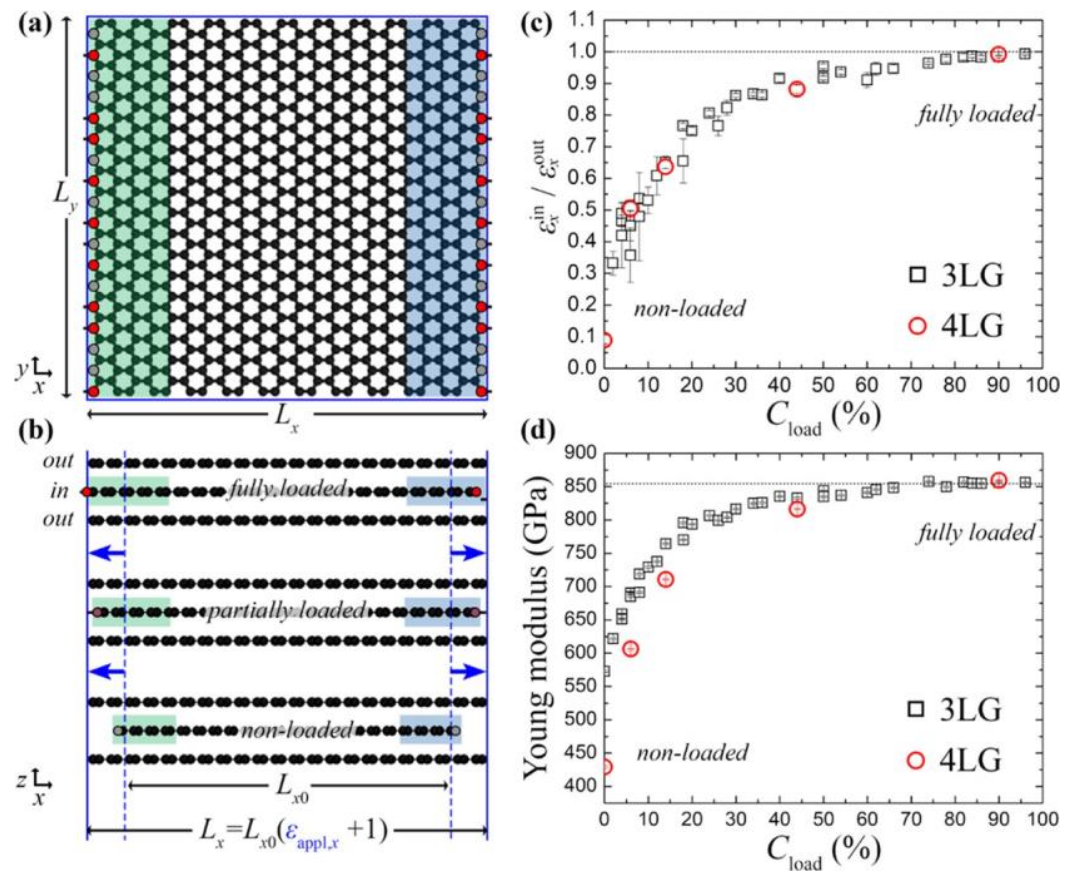

Figure 4. (a) Top-down view of an inner layer of an FLG considered in the MD simulations. The atoms in the middle area, delimited by the green and blue regions, have been set not to interact with each other to avoid the effect of periodic images. The red atoms correspond to the active sites at which the interactions with their symmetric pairs at the opposite end of the simulation box are allowed to mimic axial strain 
transfer. (b) Schematic representation of a 3LG experiencing an external uniaxial strain by $\varepsilon_{\text {appl,x }}$ with axially fully loaded (top), partially loaded (middle), and nonloaded (bottom) inner layers. The blue vertical lines depict the boundaries of the simulation box before (dashed lines) and after (solid lines) the deformation. The deformations of the inner layers have been exaggerated for clarity. (c) Stress-transfer efficiency $\varepsilon^{\text {in }}{ }_{x} / \varepsilon^{\text {out }}{ }_{x}$ and (d) the Young's modulus of $3 \mathrm{LG}(\square)$ and $4 \mathrm{LG}(\mathrm{O})$ at $\mathrm{T}=300 \mathrm{~K}$ as a function of the percentage of interactive sites, Cload.

like geometry, which employs layers of rectangular shape with the same area and exposed steps of the real flake (Table S1 in the Supporting Information), as shown in Figure S8 in the Supporting Information. As a reference case, we also report a typical nonladdered configuration (rectangular flake, Figure S9 in the Supporting Information), where we show that, as expected, by accounting only for the shear transfer mechanism, all of the load is carried by the outermost layers, while the innermost ones are nearly unstressed, having no interface with the matrix.

For the worst-case scenario represented by the absence of any transfer contribution at the edge sites of graphene flake, which is addressed with MD simulation in the next section, the ideal configuration for the optimal load transfer would comprise innermost layers bound to the matrix along both sides to achieve, due to the restrain conditions, a uniform strain on the whole flake. The flake architecture that satisfies this condition is the double-ladder configuration (Figure 1c mid panel and Figure 3b) or, in other words, a step pyramid-like architecture where the four graphene layers are stacked symmetrically. This allows to obtain a ratio between the average strain in the flakes and the applied strain at the boundary $\langle\varepsilon\rangle / \varepsilon_{\text {appl }} \cong 1$ in the whole area of all four layers, thus significantly increases the stiffness and bearing capacity of the resulting composite.

Molecular Dynamics Simulations. Upon axial loading of a multilayer graphene composite, the stress from the polymer is mainly transferred to the outer layers of the FLG. As already stated, the externally applied strain cannot be transferred effectively to the inner layers due to the following reasons: (i) the dispersive interlayer interactions are relatively weak, and thus the stress is transferred ineffectively between the adjacent layers, and (ii) the absence of direct contact (adhesion) of the inner basal planes with the polymer. The former issue can be partially overcome using a ladder geometry of the FLG, as already discussed. However, as indicated by the FEM numerical results presented above, this is not sufficient to explain the very efficient strain transfer implied by our experimental Raman observations. The effect of the latter issue is discussed in this section.

To understand the efficiency of strain transfer at the atomistic level, we performed MD simulations using the LCBOP force field $^{45}$ to describe the interatomic interactions. An advantage of atomistic MD simulations is that the dispersive pairwise interactions are modeled in an explicit manner, thereby introducing corrugations to the graphene surface that allow for finite strain transfer between the adjacent graphene sheets. The major disadvantage is the restriction on the size of the FLG that can be investigated. The MD simulations were performed on uniaxially loaded $3 \mathrm{LG}$ and $4 \mathrm{LG}$ with dimensions $12.3 \times 10.7 \mathrm{~nm}^{2}$ at room temperature (see Figure S10 in the Supporting Information). Different kinds of boundary conditions were imposed on the outer and the inner graphene layers along the strain direction to phenomenologically mimic the strain transferred from the deformed simulation box (representing the surrounding polymer matrix) to the individual graphene layers. That is, (i) the outer graphene layers experience full periodic boundary conditions (PBC) along the strain direction; hence, the strain transfer between the system and these layers is maximum. (ii) For the inner graphene layers, we devised a special kind of boundary conditions in which by adjusting the number of the active pairwise interaction sites between the edge regions located at the opposite ends of the inner layer(s) in 3LG (4LG), one can tune the strain transfer between the deformed simulation box and these layers. The latter condition accounts for the possible load transfer at edge sites due to the bonding of the matrix polymeric molecules with atoms at the edges of the inner graphene layers. The applicability of this approach rests in the assumption that the stress transfer along the normal direction is minimal and it is instead dominated by graphene edges. Recently, ${ }^{46}$ it was shown that in epoxy/graphene interfaces with sandwich-like geometries (where any edge effects are eliminated and the effect of the local intermolecular interactions is isolated), pristine graphenes drift spontaneously along the lateral directions at $300 \mathrm{~K}$, indicating zero local shear strength.

The basic assumption of the MD calculations is that the interface of polymer/outer graphene layers is ideal, like in the FEM model. As the molecules of the matrix are not simulated in our MD, we do not explicitly take into account the interaction of the polymeric molecules with the graphene sheets, which is a task of significant complexity and beyond the current scope. However, the bonding of the matrix with the edges of the inner graphene layers is effectively considered through the boundary conditions mentioned above. The FEM calculations performed on the exact geometry of the studied sample demonstrated that the area of the inner layers (Figure 3a, bottom panel), which are not in contact with the polymer matrix, can carry only a small fraction of the externally applied deformation. Besides, the experimental findings unambiguously indicate that the mechanically vulnerable part of the ladder-like samples is the $4 \mathrm{LG}$ part (see Table 1 and Figure S6), which fails at a strain of $0.75 \%$. Therefore, up to this strain level $(0.75 \%)$, the inner layers of the whole unit experience the same deformation as the outer ones, implying a good degree of bonding between the atoms located at the edges of the graphene sheets and the polymeric molecules. It is well known that graphene edges are more reactive compared to the inertness of the basal planes. ${ }^{47-50}$ Also, depending on the sample, multilayer graphene produced by mechanical exfoliation exhibits nanometer-size contact areas with the polymeric molecules as a result of the steps existing at the edges on their ends. ${ }^{51}$ Therefore, in the MD simulation we modeled the tunability of strain transfer at the inner layers through direct bonding between the edge atoms of inner graphene layers and matrix molecules by 
applying the special type of boundary conditions mentioned above. Under these conditions, the presence of covalent bonds between the randomly chosen edge atoms (active sites) located at the opposite ends of the inner sheets along the direction of uniaxial loading was permitted (Figure 4a). In this way, we can phenomenologically assess the amount of normal stress transmission through the ends. It would be worth mentioning that an alternative way to implement these boundary conditions would be to fix the positions of individual edge atoms and displace them in accordance with the deformation of the simulation box; we have performed the same tests using this approach and obtained identical results.

The efficiency of the strain transfer into the inner layers is accounted for in our MD model via the parameter $\mathrm{C}_{\text {load, }}$ which is defined as the ratio of the number of active interaction sites (atoms) to the total number of sites at the opposing edges of the inner graphene layers (see red and gray atoms for the interactive and noninteractive sites, respectively, at the edge of the inner layer depicted in Figure $4 \mathrm{a}$ ). Subsequently, $\mathrm{C}_{\text {load }}=100 \%$ accounts for axially fully loaded inner layers, i.e., all interactions at the edge sites are enabled (Figure $4 \mathrm{~b}$, top); for partially loaded inner layers, the interactions are enabled in a fraction of randomly chosen edge sites $\left(0 \%<\mathrm{C}_{\text {load }}<100 \%\right.$, Figure $4 \mathrm{~b}$, middle); for axially nonloaded inner layers, all interactions among these sites are turned off $\left(\mathrm{C}_{\text {load }}=0 \%\right.$, Figure $4 \mathrm{~b}$, bottom); hence, the deformation of the inner layers is merely originated from the dispersive interactions with the outer layers, as occurs in the FEM simulations presented in the previous section. The strain transfer efficiency in this framework is quantified by the ratio $\varepsilon^{\text {in }}{ }_{x} / \varepsilon^{\text {out }}{ }_{x}$, where $\varepsilon^{\text {in }}{ }_{x}$ and $\varepsilon^{\text {out }}{ }_{x}$ denote the strain at the central region of the inner and outer graphene layers, respectively. More details considering the estimation of $\varepsilon^{\text {in }}{ }_{x}$ and $\varepsilon^{\text {out }}{ }_{x}$ are presented in the Methods section. Consequently, $\varepsilon^{\text {in }}{ }_{x} / \varepsilon^{\text {out }}{ }_{x}=0$ means that no strain has been transferred to the inner layers, while $\varepsilon^{\text {in }}{ }_{x} / \varepsilon^{\text {out }}{ }_{x}=1$ means maximum strain transfer efficiency or in other words, the inner graphene sheets experience the same deformation as the outer ones.

Figure 4c,d present the stress transfer efficiency and the Young's modulus of 3LG and 4LG as a function of $\mathrm{C}_{\text {load, }}$, respectively. For the case of nonloaded inner layers (the absence of axial strain transfer, $\mathrm{C}_{\text {load }}=0 \%$ ), the strain is transferred to the inner layers of the FLG solely by the dispersive interlayer interactions, thus leading to minimal strain transfer efficiencies in the order of $\sim 10-20 \%$ for the external strain up to $\varepsilon_{\text {appl, }} \cong 1 \%$. This confirms the results of FEM simulation, where we find $\varepsilon_{x}^{\text {in }} / \varepsilon_{x}^{\text {out }} \cong$ $11.2 \%$ (4LG, Figure S8 in the Supporting Information). The Young's Modulus of the axially nonloaded 3LG (4LG) is about $2 / 3(2 / 4)$ of that of the single-layer graphene, suggesting that the inner layers of the axially nonloaded FLG cannot sustain deformations. The inability of the inner layers to carry loads was also quantified by the computed stress of the inner layers during the course of the simulation, which assumed small positive values of the order of $0.5 \mathrm{GPa}$, while the stress of the outer layers increases continuously (see Figure S11 in the Supporting Information). By increasing the coupling of the inner layers with the surrounding polymer matrix, namely, increase the number of active sites (partially loaded FLG, $0 \%<\mathrm{C}_{\text {load }}<100 \%$ ), both $\varepsilon^{\text {in }}{ }_{x} / \varepsilon^{\text {out }}{ }_{x}$ and the Young's modulus increase as shown in Figure $4 \mathrm{c}, \mathrm{d}$, respectively. It is shown that already a bonding of $40 \%$ of the edge atoms results in a high strain transfer efficiency of about $90 \%$ (Figure 4c).

Finally, for the ideal case of axially fully loaded inner layers $\left(\mathrm{C}_{\text {load }}=100 \%\right)$, our MD simulations predict that the Young's moduli of $3 \mathrm{LG}$ and $4 \mathrm{LG}$ at $\mathrm{T}=300 \mathrm{~K}$ are the same, i.e., $\sim 855 \pm 10 \mathrm{GPa}$. For comparison, in the small strain regime $(\varepsilon<1 \%$ ), the Young's modulus of 1LG was calculated to be $840 \pm 20 \mathrm{GPa}$ at $300 \mathrm{~K}$, suggesting that when the strain is transferred in an efficient manner, the mechanical properties of the fully loaded FLG are similar to those of 1LG. Also, in conjunction with the reported FEM simulations, we suggest that the experimental results obtained from the complex ladder architecture cannot be solely interpreted by a shear transfer mechanism between the polymer and the various portions of multilayer graphene, but a certain degree of normal/axial stress transmission occurs through the bonding of the graphene ends with the polymer matrix.

The scaling-up of such flake architectures is a challenge in the field of 2D materials. Recently, the CVD syntheses of about $100 \mu \mathrm{m}$ pyramid-like $2 \mathrm{LG}$ and $3 \mathrm{LG}$ domains with hexagonal shapes on $\mathrm{Cu}$ foils was reported. ${ }^{52}$ Moreover, CVD can easily produce pyramid-like structures of $2 \mathrm{LG}$ and thicker flakes of transition metal dichalcogenides, although the thickness controllability is still a challenging task ${ }^{53-55}$ Importantly, pyramid-like heterostructures have also been realized, while further optimization is necessary to control flake size and thickness. ${ }^{56}$ Therefore, our approach could be applied in the future in a diversity of devices based on strain engineering of $2 \mathrm{D}$ crystals due to the efficiency of strain transfer to all involved layers.

\section{CONCLUSIONS}

Previous studies have shown that few-layer graphene flakes embedded in composites under axial loadings (uniaxial or biaxial) are deformed inefficiently due to incomplete stress transfer from the outer layers to the inner layers of the assembly. This is clearly manifested by the fact that the $2 \mathrm{D}$ and $\mathrm{G}$ phonon positions under strain tend to decrease with the increase of graphene thickness. Due to this phenomenon, fewlayer flakes produced by exfoliation are inferior as reinforcing agents in nanocomposites compared to monolayers and bilayers.

In the present study, we show that few-layer graphene can be stressed efficiently by adopting graphene flakes with particular morphology. Herein, a graphene flake in a ladder-like form, which consists of mono-, bi-, tri-, and four-layer graphene 
consecutively, was tested under tension using Raman spectroscopy. In this form, the stress transfer mechanism to the middle layers differs from the conventional shear mechanism through the weak vdW bonds, while each individual monolayer is stretched directly from the surrounding polymer. The phonon strain rates of the 3LG and 4LG parts of the flake are found to be similar to those of 1LG and 2LG. A step pyramidlike architecture is proposed as an ideal form for efficient loading of layered materials, having a sufficient transfer length for the stress build-up from both edges of the flake.

The numerical results from MD and FEM simulations suggest that in the absence of inner layer-polymer coupling (FLG with axially nonloaded inner layers), the strain transfer from the outer to the inner graphene layers is very weak, in the order of $\sim 10 \%$ of the applied strain at the composite boundary. Furthermore, our MD simulations show that the Young's modulus of 3LGs and 4LGs with axially nonloaded inner layers is approximately $2 / \mathrm{N}_{\mathrm{L}}$ times to that of $1 \mathrm{LG}$, where $\mathrm{N}_{\mathrm{L}}$ is the number of layers, suggesting that in this case, the inner layers cannot carry a substantial load, thus significantly affecting the mechanical properties of the composite as $\mathrm{N}_{\mathrm{L}}$ increases. However, by increasing the axial coupling between the polymer and the inner graphene layers, both the strain transfer efficiency and the Young's modulus are improved substantially. When the inner layers are fully loaded axially, then the strain transfer efficiency becomes $100 \%$ and the Young's modulus of FLG becomes close to those of 1 LGs.

In practical terms, the edge coupling through direct molecular bonding with the matrix is not controllable. On the other hand, using FEM simulations, we have demonstrated that an almost full loading of the inner layers can be equivalently realized by a flake geometry laddered on both edges along the direction of load application. In principle, multiaxial states of load would require the realization of an isotropic laddering, i.e., along all directions, in a step pyramidlike architecture. It can thus be concluded that FLGs with geometries that increase the interface between the inner layers and the polymer matrix display enhanced mechanical properties due to the optimal load transfer, opening the opportunity to exploit the full potential of FLGs as reinforcing fillers in nanocomposites.

\section{METHODS}

Sample Preparation. A poly(methyl methacrylate) PMMA beam $(\sim 3 \mathrm{~mm}$ in thickness, $\sim 12 \mathrm{~cm}$ in length, and $\sim 1.1 \mathrm{~cm}$ in width), supplied form S. Varnakiotis Glass S.A., spin-coated ( 4000 rpm) with a thin ( 200 nm) layer of epoxy-based photoresist SU-8 (2000.5 Microchem) was employed as the substrate to increase the optical contrast of the flake. Curing of the SU-8 follows three steps: (a) soft bake process at $\sim 90{ }^{\circ} \mathrm{C}$ for $10 \mathrm{~min}$; (b) UV radiation with a wavelength of $\sim 302 \mathrm{~nm}\left(3.5 \mathrm{~mW}\right.$, UVP Upland) exposed for $10 \mathrm{~s}$ and (c) postbake at $\sim 100{ }^{\circ} \mathrm{C}$ for $5 \mathrm{~min}$. The deposition of the graphene occurred directly on the polymer by mechanical cleaving of the highly ordered pyrolytic graphite (HOPG) bulk crystals supplied by Graphene Supermarket using the scotch tape method. A conventional 3M scotch tape is used. The peeling process is aimed to have large shiny flat graphite domains, 3-4 millimeters in size. This is beneficial to obtain large ladder-like graphene flakes. The tape is deposited as flat as possible to avoid trapped air in the tape/substrate interface. After the deposition, the tape remains on top of the beam for a couple of minutes to ensure better contact between the graphene and the polymer. Finally, the tape was removed quite slowly at low angles, giving rise to significant shear stress at the tape/graphene interface.

Raman Spectroscopy. The desired graphene flakes were identified using optical microscopy, and the number of layers was determined by the line-shape of the 2D Raman peak. Afterward, another thin layer of PMMA (495 PMMA A3 Microchem) was spincoated on top with a speed of $\sim 6000 \mathrm{rpm}$, resulting in a thickness of $\sim 100 \mathrm{~nm}$. Based on the frequency of the G Raman peak at rest, the procedure induced minimal residual strain to the sample.

The fully embedded sample was tested mechanically under a Raman microscope. Raman spectra were collected in the backscattering geometry using a Renishaw InVia 2000 spectrometer equipped with 2400 grooves/mm having a spectral resolution of $\sim 2.5 \mathrm{~cm}^{-1}$ and spectral accuracy of $\sim 0.1 \mathrm{~cm}^{-1}$. For excitation, the $514.5 \mathrm{~nm}$ line of an $\mathrm{Ar}^{+}$laser was focused on the sample by means of an 100× objective lens (NA $=0.85$ ). The polarization of the incident light was kept parallel to the applied strain axis. The Raman mapping took place with a step size of $6 \mu \mathrm{m}$, using a high speed optically encoded motorized sample stage (Renishaw, U.K.). The phonon frequencies were obtained by fitting Lorentzian functions to the experimental data, after background subtraction.

Strain Device. A four-point-bending testing apparatus was employed to conduct tensile measurements. The level of tensile strain on the top surface of the beam is monitored by controlling the applied deflection in the center of the beam. The increment step of strain was $0.1 \%$. The strain $\varepsilon$ is related to deflection $\delta$, the length of the span L, and the thickness t of the PMMA bar according to the equation: $\varepsilon \delta()=4.47 \_\delta_{2}^{t}$. This relation has been validated by

$L$ independent measurements taken with a strain gauge attached in the middle area of the beam, where the Raman spectra are collected. More details about the four-point bending apparatus can be found in refs 19,21.

Finite Element Simulations. The FEM model of the experimentally studied flake of Figure 1a is shown in Figure S7 in the Supporting Information. The rectangular nonladdered configuration (Figure S9 in the Supporting Information) was constructed for comparison by keeping the same area of the biggest layer (nr. 1) of the experimental flake and with an aspect ratio given by the ratio of the principal axes of inertia of the layer area. The single-side-ladder configuration (Figure S8 in the Supporting Information) is derived from the rectangular configuration by keeping for the four layers the same areas of the real case. In this way, we also obtain similar ladder steps with respect to the experimentally 
investigated flake. The double-laddered configuration (ideal case, Figure $3 b$ ) is the mirrored version of the single-laddered configuration by keeping the area of layer 4 constant (i.e., the smaller and uppermost layer). Dimensions of the different flakes studied are reported in Table $\mathrm{S} 1$ in the Supporting Information.

The PMMA and SU-8 matrixes are modeled with solid elements and linear elastic and isotropic material law (Young's moduli of 2.5 and 4.0 GPa and corresponding Poisson's ratios of 0.375 and 0.22 , respectively). Graphene layers are represented via fully integrated shell elements $(2 \times 2$ Gauss points) based on the Reissner-Mindlin kinematic assumption, with thickness assumed conventionally of $0.3415 \mathrm{~nm}$. The constitutive law for graphene is assumed to be linear elastic and isotropic with $\mathrm{E}=1 \mathrm{TPa}$ and $\mathrm{v}=0.2$.

The interface interaction between graphene sheets is described via an equivalent continuum cohesive model, derived according to the work by Jiang et al. ${ }^{57}$ Graphene layers and polymer matrixes are assumed to be perfectly bonded. The weak interlayer bonding between graphene flakes due to vdW interactions can be represented by the following Lennard-Jones 6-12 potential

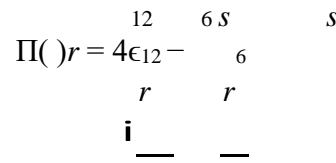

with $\mathrm{r}$

$$
\text { being the spatial distance between an atom pair, }{ }^{j}{ }_{k} \mathrm{yz}_{\{s}=0.34556
$$

$\mathrm{nm}$

is the interparticle distance at which the potential is zero, $h=2 s$ is the equilibrium distance between the two atoms, and $\epsilon=2.39 \mathrm{meV}$ is the corresponding bond energy. ${ }^{58}$ Considering the two layers, the homogenized cohesive energy per unit area, equivalent to the discrete weak

$$
\begin{aligned}
& \begin{array}{rlllll}
\sum^{\text {pair }} & 2 \int_{0}^{\infty} & 2 & 2 & \frac{2 s^{10}}{5 r^{10}} & \frac{s^{4}}{r^{4}} \quad \begin{array}{l}
\text { vdW interactions, can be calculated from the sum } \\
\text { pairs }
\end{array} \\
\Phi() r=2 \pi \psi \quad \Pi() r z z \mathrm{~d}=2 \pi \psi \epsilon s-
\end{array} \\
& \sqrt{3} l_{0}^{2} \quad i^{i=1} \mathrm{ij}_{\mathrm{k}} \quad \mathrm{yz}_{\{} \quad(2)
\end{aligned}
$$

where $\psi=\Gamma / 3$ is a homogenization parameter representing the number of atoms per unit area, 10 is the equilibrium $\mathrm{C}-\mathrm{C}$ bond length $(0.142$ $\mathrm{nm}$ ) before deformation, and $\Gamma=4$ for the hexagonal lattice. The cohesive energy is weighted at each node of the mesh, assuming that the area of influence is defined by the space enclosed within the centroids of the adjacent finite elements. The equilibrium distance hG/G between graphene sheets can be derived by imposing $\frac{\partial \Phi}{\partial r}=0$, obtaining h]s, which is the initial distance of graphene layers adopted in the FEM simulations. Introducing tangential and normal perturbation displacements at the interface, denoted as $\mathrm{u}$ and $\mathrm{v}$, respectively, the normal stress and shear cohesive stress modeling the vdW interactions between the graphene layers (Figure S7 in the Supporting Information) are then determined as follows

$$
\begin{aligned}
\sigma_{\mathrm{vdW}} & =\frac{\left.\partial \Phi\right|_{r=h+v}}{\partial v}=8 \pi \psi^{2} \epsilon s_{\mathbf{j}} \frac{2 s^{5}}{5(h+v)^{5}}-\frac{s^{11}}{(h+v)^{11}} \mathrm{y} \\
\tau_{\mathrm{vdW}} & =\frac{\left.\partial \Phi\right|_{r=h+v}}{\partial u}=0
\end{aligned}
$$

Positive values of $\sigma_{\mathrm{vdW}}$ and $\mathrm{v}$ denote traction and separation displacements, respectively. It can be easily computed that the interface energy vanishes starting from $\mathrm{v} \approx 3 \mathrm{~h}$. Thus, in our case, the interface interaction is implemented between each of the 4 layers of the flake.

Molecular Dynamics Simulations. The MD simulations of $3 \mathrm{LG}$ and $4 \mathrm{LG}$ were performed using the LAMMPS simulation package ${ }^{59}$ with a time step of $0.0008 \mathrm{ps}$, at room temperature (pressure $1 \mathrm{~atm}$ ) using the Nose'- Hoover thermostat (barostat) and an effective relaxation time of $0.1 \mathrm{ps}(1 \mathrm{ps}){ }^{60-62}$ The LCBOP force field is employed to describe the C-C interactions, ${ }^{45}$ which has been shown ${ }^{63,64}$ to provide qualitatively similar results with other relevant pairwise force fields ${ }^{65,66}$ as well as phonon dispersion curves in very good agreement with the available experimental data.${ }^{67}$ Each graphene layer consists of 5000 atoms, with a size of $\sim 12.3 \times 10.7 \mathrm{~nm}^{2}$, as shown in Figure S10 in the Supporting Information. Along the lateral dimensions ( $\mathrm{x}, \mathrm{y}$ ) of the FLG, PBC are applied in the simulation box, while along the normal (z) direction, the boundaries are aperiodic. The equilibrium $\mathrm{C}-\mathrm{C}$ bond length for graphene is $\sim 0.142 \mathrm{~nm}$, while the interlayer distance for FLG is calculated to be $\mathrm{h}=0.341 \mathrm{~nm}$ at $\mathrm{T}=300 \mathrm{~K}$ for this force field.

The MD simulations consist of three phases. (i) Initially, the samples are left to relax for 80 ps within the $N \sigma_{x x} \sigma_{y y} L_{z} T$ ensemble [constant number of atoms $(\mathrm{N})$, lateral stress $\left(\sigma_{\mathrm{xx}}, \sigma_{\mathrm{yy}}\right)$, constant box length along the normal direction $\left(\mathrm{L}_{\mathrm{z}}\right)$ and temperature $(\mathrm{T})$ ] to achieve their corresponding equilibrium values. (ii) Then, the samples are simulated for 40 ps to compute their equilibrium time-averaged dimensions. (iii) At the last phase of the simulation, the equilibrated FLGs are deformed uniaxially along the armchair direction with a constant strain rate upon increasing the $\mathrm{x}$ component of the simulation box. The strain rate was set to $\varepsilon$ appi, $\mathrm{x}=12.5 \times 10^{-4} \mathrm{ps}^{-1}$ for uniaxial deformations up to $2 \%$; varying the applied strain rate using a 10 times smaller rate granted similar results. Note that, during the deformation phase, the barostat is switched off along the strain axis; hence, the sampling is performed using the $\mathrm{N} \varepsilon_{\mathrm{xx}} \sigma_{\mathrm{yy}} \mathrm{L}_{\mathrm{z}} \mathrm{T}$ statistical ensemble. The strain transfer efficiency 
was estimated as the ratio $\varepsilon^{\mathrm{in}}{ }_{\mathrm{x}} / \varepsilon^{\text {out }}$ in the small strain regime $\left(\varepsilon_{\mathrm{appl}, \mathrm{x}}<1 \%\right)$. $\varepsilon^{\mathrm{in}_{\mathrm{x}}}$ was spatially averaged over a $5 \times 2 \mathrm{~nm}^{2}$ area at the central region of the inner graphene layers (see Figure 4a).

The stress of the individual layers is computed from the symmetric per-atom Cauchy stress tensor (the sum of the kinetic and the configurational part), which is given by the following equation: ${ }^{68}$

$\tau i, \alpha \beta=-\_V 1 i$ ÅÄÇmu $u i, \alpha \beta i,+\frac{1}{2} \sum_{i N_{i}^{p a r}}\left(r F_{i, \alpha}\right.$ ipair,$\left.\beta+r F_{j, \alpha j \text { pair }, \beta}\right) \tilde{N} O ̈ E ́$ for the pair style

force field employed here. ${ }^{45}$ This formula computes the six components of the symmetric per-atom tensor, where $\mathrm{V}_{\mathrm{i}}$ is the volume occupied by the ith atom, $\alpha, \beta$ denotes spatial directions, and $\mathrm{i}, \mathrm{j}$ denotes atoms. The first term of the equation is the ideal gas contribution to the peratom stress tensor. The second term is the sum of the pairwise energy contribution over all of the $\mathrm{N}_{\mathrm{i}}$ pair interacting neighbors of the ith atom, while $\mathrm{r}_{\mathrm{i}, \alpha}, \mathrm{r}_{\mathrm{j}, \alpha}$ denotes the positions and $\mathrm{F}^{\mathrm{pai}} \mathrm{i}_{\mathrm{i}, \beta}, \mathrm{F}^{\text {pair }}{ }_{\mathrm{j}, \beta}$ denotes the forces of the interacting atoms. It should be noted that since a pairwise force field was used, the covalent and the long-range interactions are described collectively by the pairwise terms. The stress of a collection $\mathrm{k}$ of particles (e.g., a group of atoms comprising a specific layer) was obtained through the following

expression: ${ }^{69} \tau_{k,} \quad=\frac{1}{-} \sum_{j \in k} V \tau_{\alpha \beta V k j k, \alpha \beta}$, where $\mathrm{V}_{\mathrm{k}}$ is the total volume occupied by the collection of atoms and $\mathrm{j}$ denotes atoms belonging to the collection $k$. In our case, $V_{k}$ equals the instantaneous volume of the individual $k$ th graphene layer $\left(V_{k}=l^{k} \cdot l^{k} \cdot h\right)$ and is recomputed at each step of the simulation.

\section{ASSOCIATED CONTENT}

* Supporting Information

The Supporting Information is available free of charge at https://pubs.acs.org/doi/10.1021/acsami.0c18774.

Brightness profile and Raman mapping of the studied graphene flake (S1); uniaxial Raman response of monolayer graphene (S2); uniaxial Raman response of bilayer graphene (S3); uniaxial Raman response of trilayer graphene (S4); uniaxial Raman response under compression of trilayer graphene (S5); uniaxial Raman response of four-layer graphene (S6); finite element simulations (S7); molecular dynamic simulations (S8)

(PDF)

\section{AUTHOR INFORMATION}

Corresponding Author

Konstantinos Papagelis - Institute of Chemical Engineering

Sciences, Foundation for Research and Technology-Hellas

(FORTH/ICE-HT), Patras 26504, Greece; School of

Physics, Department of Solid State Physics, Aristotle

University of Thessaloniki, Thessaloniki 54124, Greece; ㅇ orcid.org/0000-0001-5094-9837; Email: kpapag@

physics.auth.gr

Authors

Aristotelis P. Sgouros - School of Chemical Engineering,

National Technical University of Athens (NTUA), Athens

15780, Greece; 이이.org/0000-0003-4183-686X

Charalampos Androulidakis - Institute of Chemical

Engineering Sciences, Foundation for Research and

Technology-Hellas (FORTH/ICE-HT), Patras 26504,

Greece; $\odot$ orcid.org/0000-0002-5264-1841

Georgia Tsoukleri - Institute of Chemical Engineering

Sciences, Foundation for Research and Technology-Hellas

(FORTH/ICE-HT), Patras 26504, Greece 
George Kalosakas - Department of Materials Science, University of Patras, Patras 26504, Greece

Nikos Delikoukos - Institute of Chemical Engineering

Sciences, Foundation for Research and Technology-Hellas

(FORTH/ICE-HT), Patras 26504, Greece; $\odot$ orcid.org/

0000-0002-4277-734X

Stefano Signetti - Laboratory of Bio-Inspired, Bionic, Nano,

Meta Materials \& Mechanics, Department of Civil,

Environmental and Mechanical Engineering, University of

Trento, I-38123 Trento, Italy; ๑ orcid.org/0000-0003-

4128-0953

Nicola M. Pugno - Laboratory of Bio-Inspired, Bionic, Nano,

Meta Materials \& Mechanics, Department of Civil,

Environmental and Mechanical Engineering, University of

Trento, I-38123 Trento, Italy; School of Engineering and

Materials Science, Queen Mary University of London, London

E1 4NS, United Kingdom; ๑ orcid.org/0000-0003-2136-

2396

John Parthenios - Institute of Chemical Engineering Sciences,

Foundation for Research and Technology-Hellas (FORTH/ ICE-HT), Patras 26504, Greece; ๑ orcid.org/0000-0001-

6066-7120

Costas Galiotis - Institute of Chemical Engineering Sciences,

Foundation for Research and Technology-Hellas (FORTH/

ICE-HT), Patras 26504, Greece; Department of Chemical

Engineering, University of Patras, Patras 26504, Greece; ๑ orcid.org/0000-0001-8079-5488

Complete contact information is available at: https://pubs.acs.org/10.1021/acsami.0c18774

Author Contributions

This manuscript was written through contributions of all authors. All authors have given approval to the final version of the manuscript.

Notes

The authors declare no competing financial interest.

\section{ACKNOWLEDGMENTS}

NMP is supported by the European Commission under the Graphene Flagship Core 3 grant No. 881603 (WP14 "Composites").

\section{REFERENCES}

(1) Samorì, P.; Kinloch, I. A.; Feng, X.; Palermo, V. Graphene-Based Nanocomposites for Structural and Functional Applications: Using 2Dimensional Materials in a 3-Dimensional World. 2D Mater. 2015, 2, No. 030205.

(2) Backes, C.; Abdelkader, A. M.; Alonso, C.; Nolan, H. Production and Processing of Graphene and Related Materials. 2D Mater. 2020, 7, 1-282.

(3) Kim, K.; Coh, S.; Tan, L. Z.; Regan, W.; Yuk, J. M.; Chatterjee, E.; Crommie, M. F.; Cohen, M. L.; Louie, S. G.; Zettl, A. Raman Spectroscopy Study of Rotated Double-Layer Graphene: Misorientation-Angle Dependence of Electronic Structure. Phys. Rev. Lett. 2012, 108, No. 246103.

(4) Bao, W.; Jing, L.; Velasco, J.; Lee, Y.; Liu, G.; Tran, D.; Standley, B.; Aykol, M.; Cronin, S. B.; Smirnov, D.; Koshino, M.; McCann, E.; Bockrath, M.; Lau, C. N. Stacking-Dependent Band Gap and Quantum Transport in Trilayer Graphene. Nat. Phys. 2011, No. 948.

(5) Androulidakis, C.; Koukaras, E. N.; Paterakis, G.; Trakakis, G.; Galiotis, C. Tunable Macroscale Structural Superlubricity in twoLayer Graphene via Strain Engineering. Nat. Commun. 2020, 11, No. 1595. 
(6) Cao, Y.; Fatemi, V.; Fang, S.; Watanabe, K.; Taniguchi, T.; Kaxiras, E.; Jarillo-Herrero, P. Unconventional Superconductivity in Magic-Angle graphene Superlattices. Nature 2018, 556, 43-50.

(7) Yankowitz, M.; Chen, S.; Polshyn, H.; Zhang, Y.; Watanabe, K.; Taniguchi, T.; Graf, D.; Young, A. F.; Dean, C. R. Tuning Superconductivity in Twisted Bilayer Graphene. Science 2019, No. eaav1910.

(8) Signetti, S.; Taioli, S.; Pugno, N. M. 2D Material Armors Showing Superior Impact Strength of Few Layers. ACS Appl. Mater. Interfaces 2017, 9, 40820-40830.

(9) Zhu, S.; Li, T. Hydrogenation Enabled Scrolling of Graphene. J. Phys. D: Appl. Phys. 2013, 46, No. 075301.

(10) Sgouros, A.; Sigalas, M. M.; Papagelis, K.; Kalosakas, G. Transforming Graphene Nanoribbons into Nanotubes by use of Point Defects. J. Phys.: Condens. Matter 2014, 26, No. 125301.

(11) Sgouros, A. P.; Kalosakas, G.; Sigalas, M. M.; Papagelis, K. Exotic Carbon Nanostructures Obtained through Controllable Defect Engineering. RSC Adv. 2015, 5, 39930-39937.

(12) Papageorgiou, D. G.; Kinloch, I. A.; Young, R. J. Mechanical Properties of Graphene and Graphene-Based Nanocomposites. Prog. Mater. Sci. 2017, 90, 75-127.

(13) Du, J.; Pei, S.; Ma, L.; Cheng, H. M. 25th Anniversary Article: Carbon Nanotube- and Graphene-Based Transparent Conductive Films for Optoelectronic Devices. Adv. Mater. 2014, 26, 1958-1991. (14) Tsoukleri, G.; Parthenios, J.; Papagelis, K.; Jalil, R.; Ferrari, A. C.; Geim, A. K.; Novoselov, K. S.; Galiotis, C. Subjecting a Graphene Monolayer to Tension and Compression. Small 2009, 5, $2397-2402$.

(15) Xu, P.; Kang, J.; Suhr, J.; Smith, J. P.; Booksh, K. S.; Wei, B.; Yu, J.; Li, F.; Byun, J.-H.; Oh, Y.; Chou, T.-W. Spatial Strain Variation of Graphene Films for Stretchable Electrodes. Carbon 2015, 93, 620- 624.

(16) Xu, C.; Xue, T.; Guo, J.; Qin, Q.; Wu, S.; Song, H.; Xie, H. An Experimental Investigation on the Mechanical Properties of the Interface Between Large-Sized Graphene and a Flexible Substrate. J. Appl. Phys. 2015, 117, No. 164301.

(17) Li, Z.; Kinloch, I. A.; Young, R. J.; Novoselov, K. S.; Anagnostopoulos, G.; Parthenios, J.; Galiotis, C.; Papagelis, K.; Lu, C.-Y.; Britnell, L. Deformation of Wrinkled Graphene. ACS Nano 2015, 9, 3917-3925.

(18) Androulidakis, C.; Koukaras, E. N.; Parthenios, J.; Kalosakas, G.; Papagelis, K.; Galiotis, C. Graphene Flakes under Controlled Biaxial Deformation. Sci. Rep. 2015, 5, No. 18219.

(19) Androulidakis, C.; Koukaras, E. N.; Frank, O.; Tsoukleri, G.; Sfyris, D.; Parthenios, J.; Pugno, N.; Papagelis, K.; Novoselov, K. S.; Galiotis, C. Failure Processes in Embedded Monolayer Graphene under Axial Compression. Sci. Rep. 2014,4 , No. 5271.

(20) Jiang, T.; Huang, R.; Zhu, Y. Interfacial Sliding and Buckling of Monolayer Graphene on a Stretchable Substrate. Adv. Funct. Mater. 2014, 24, 396-402.

(21) Anagnostopoulos, G.; Androulidakis, C.; Koukaras, E. N.; Tsoukleri, G.; Polyzos, I.; Parthenios, J.; Papagelis, K.; Galiotis, C. Stress Transfer Mechanisms at the Submicron Level for Graphene/ Polymer Systems. ACS Appl. Mater. Interfaces 2015, 7, 4216-4223.

(22) Gong, L.; Kinloch, I. A.; Young, R. J.; Riaz, I.; Jalil, R.; Novoselov, K. S. Interfacial Stress Transfer in a Graphene Monolayer Nanocomposite. Adv. Mater. 2010, 22, 2694-2697.

(23) Gong, L.; Young, R. J.; Kinloch, I. A.; Haigh, S. J.; Warner, J. H.; Hinks, J. A.; Xu, Z.; Li, L.; Ding, F.; Riaz, I.; Jalil, R.; Novoselov, K. S. Reversible Loss of Bernal Stacking During the Deformation of Few-Layer Graphene in Nanocomposites. ACS Nano 2013, 7, 72877294.

(24) Gong, L.; Young, R. J.; Kinloch, I. A.; Riaz, I.; Jalil, R.; Novoselov, K. S. Optimizing the Reinforcement of Polymer-Based Nanocomposites by Graphene. ACS Nano 2012, 6, 2086-2095.

(25) Young, R. J.; Gong, L.; Kinloch, I. A.; Riaz, I.; Jalil, R.; Novoselov, K. S. Strain Mapping in a Graphene Monolayer Nanocomposite. ACS Nano 2011, 5, 3079-3084.

(26) Frank, O.; Tsoukleri, G.; Parthenios, J.; Papagelis, K.; Riaz, I.; Jalil, R.; Novoselov, K. S.; Galiotis, C. Compression Behavior of Single-Layer Graphenes. ACS Nano 2010, 4, 3131-3138.

(27) Androulidakis, C.; Koukaras, E. N.; Rahova, J.; Sampathkumar, K.; Parthenios, J.; Papagelis, K.; Frank, O.; Galiotis, C. Wrinkled FewLayer Graphene as Highly Efficient Load Bearer. ACS Appl. Mater. Interfaces 2017, 9, 26593-26601.

(28) Li, Z.; Kinloch, I. $\quad$ A.; Young, R. $\quad$ J.; Novoselov, $\quad$ K. $\quad$ S.; Anagnostopoulos, G.; Parthenios, J.; Galiotis, C.; Papagelis, K.; Lu, C. Y.; Britnell, L. Deformation of Wrinkled Graphene. ACS Nano 2015, 9, 3917-3925.

(29) Tsoukleri, G.; Parthenios, J.; Galiotis, C.; Papagelis, K. Embedded Trilayer Graphene Flakes under Tensile and Compressive Loading. 2D Mater. 2015, 2, No. 024009.

(30) Tripathi, M.; Awaja, F.; Paolicelli, G.; Bartali, R.; Iacob, E.; Valeri, S.; Ryu, S.; Signetti, S.; Speranza, G.; Pugno, N. M. Tribological Characteristics of Few-Layer Graphene over Ni Grain and Interface Boundaries. Nanoscale 2016, 8, 6646-6658.

(31) Frank, O.; Bousa, M.; Riaz, I.; Jalil, R.; Novoselov, K. S.; Tsoukleri, G.; Parthenios, J.; Kavan, L.; Papagelis, K.; Galiotis, C. Phonon and Structural Changes in Deformed Bernal Stacked Bilayer Graphene. Nano Lett. 2012, 12, 687-693.

(32) Geim, A. K.; Grigorieva, I. V. Van der Waals Heterostructures. Nature 2013, 499, 419.

(33) Liu, Y.; Weiss, N. O.; Duan, X.; Cheng, H.-C.; Huang, Y.; Duan, X. Van der Waals Heterostructures and Devices. Nat. Rev. Mater. 2016, 1, No. 16042.

(34) Novoselov, K. S.; Mishchenko, A.; Carvalho, A.; Castro Neto, A. H. 2D Materials and van der Waals Heterostructures. Science 2016, 353, No. aac9439.

(35) Kumar, H.; Dong, L.; Shenoy, V. B. Limits of Coherency and Strain Transfer in Flexible 2D van der Waals Heterostructures: Formation of Strain Solitons and Interlayer Debonding. Sci. Rep. 2016, 6, No. 21516.

(36) Liu, H. N.; Cong, X.; Lin, M. L.; Tan, P. H. The Intrinsic Temperature-Dependent Raman Spectra of Graphite in the Temperature Range from 4 K to 1000 K. Carbon 2019, 152, 451-458. 
(37) Ferrari, A. C.; Bonaccorso, F.; Fal'ko, V.; Novoselov, K. S.; Roche, S.; Bøggild, P.; Borini, S.; Koppens, F. H. L.; Palermo, V.; Pugno, N.; Garrido, J. A.; Sordan, R.; Bianco, A.; Ballerini, L.; Prato, M.; Lidorikis, E.; Kivioja, J.; Marinelli, C.; Ryhanen, T.; Morpurgo, A.; Coleman, J. N.; Nicolosi, V.; Colombo, L.; Fert, A.; GarciaHernandez, M.; Bachtold, A.; Schneider, G. F.; Guinea, F.; Dekker, C.; Barbone, M.; Sun, Z.; Galiotis, C.; Grigorenko, A. N.; Konstantatos, G.; Kis, A.; Katsnelson, M.; Vandersypen, L.; Loiseau, A.; Morandi, V.; Neumaier, D.; Treossi, E.; Pellegrini, V.; Polini, M.; Tredicucci, A.; Williams, G. M.; Hee Hong, B.; Ahn, J.-H.; Min Kim, J.; Zirath, H.; van Wees, B. J.; van der Zant, H.; Occhipinti, L.; Di Matteo, A.; Kinloch, I. A.; Seyller, T.; Quesnel, E.; Feng, X.; Teo, K.; Rupesinghe, N.; Hakonen, P.; Neil, S. R. T.; Tannock, Q.; Löfwander, T.; Kinaret, J. Science and Technology Roadmap for Graphene, Related twoDimensional Crystals, and Hybrid Systems. Nanoscale 2015, 7, 4598-4810.

(38) Novoselov, K. S.; Fal'ko, V. I.; Colombo, L.; Gellert, P. R.; Schwab, M. G.; Kim, K. A Roadmap for Graphene. Nature 2012, 490, 192-200.

(39) Ni, Z. H.; Yu, T.; Lu, Y. H.; Wang, Y. Y.; Feng, Y. P.; Shen, Z. X. Uniaxial Strain on Graphene: Raman Spectroscopy Study and Band-Gap Opening. ACS Nano 2008, 2, 2301-2305.

(40) Gong, L.; Young, R. J.; Kinloch, I. A.; Riaz, I.; Jalil, R.; Novoselov, K. S. Optimizing the Reinforcement of Polymer-Based Nanocomposites by Graphene. ACS Nano 2012, 6, 2086-2095.

(41) Mohiuddin, T. M. G.; Lombardo, A.; Nair, R. R.; Bonetti, A.; Savini, G.; Jalil, R.; Bonini, N.; Basko, D. M.; Galiotis, C.; Marzari, N.; Novoselov, K. S.; Geim, A. K.; Ferrari, A. C. Uniaxial Strain in Graphene by Raman Spectroscopy: G Peak Splitting, Gruneisen Parameters, and Sample Orientation. Phys. Rev. B 2009, 79, No. 205433.

(42) Huang, M.; Yan, H.; Chen, C.; Song, D.; Heinz, T. F.; Hone, J. Phonon Softening and Crystallographic Orientation of Strained Graphene Studied by Raman Spectroscopy. Proc. Natl. Acad. Sci. U.S.A. 2009, 106, 7304-7308.

(43) Androulidakis, C.; Koukaras, E. N.; Hadjinicolaou, M.; Galiotis, C. Non-Eulerian Behavior of Graphitic Materials under Compression. Carbon 2018, 138, 227-233.

(44) Androulidakis, C.; Sourlantzis, D.; Koukaras, E. N.; Manikas, A. C.; Galiotis, C. Stress-Transfer from Polymer Substrates to Monolayer and Few-Layer Graphenes. Nanoscale Adv. 2019, 1, 4972-4980.

(45) Los, J. H.; Fasolino, A. Intrinsic Long-Range Bond-Order Potential for Carbon: Performance in Monte Carlo Simulations of Graphitization. Phys. Rev. B 2003, 68, No. 024107.

(46) Kallivokas, S. V.; Sgouros, A. P.; Theodorou, D. N. Kinetic Concepts and Local Failure in the Interfacial Shear Strength of EpoxyGraphene Nanocomposites. Phys. Rev. E 2020, 102, No. 030501.

(47) Park, J.; Yan, M. Covalent Functionalization of Graphene with Reactive Intermediates. Acc. Chem. Res. 2013, 46, $181-189$.

(48) Dubois, S. M. M.; Lopez-Bezanilla, A.; Cresti, A.; Triozon, F.; Biel, B.; Charlier, J. C.; Roche, S. Quantum Transport in Graphene Nanoribbons: Effects of Edge Reconstruction and Chemical Reactivity. ACS Nano 2010, 4, No. 1971.

(49) Sharma, R.; Baik, J. H.; Perera, C. J.; Strano, M. S. Anomalously Large Reactivity of Single Graphene Layers and Edges Toward Electron Transfer Chemistries. Nano Lett. 2010, 10, 398-405.

(50) Bellunato, A.; Arjmandi Tash, H.; Cesa, Y.; Schneider, G. H. Chemistry at the Edge of Graphene. ChemPhysChem 2016, 17, No. 785.

(51) Tripathi, M.; Awaja, F.; Bizao, R. A.; Signetti, S.; Iacob, E.; Paolicelli, G.; Valeri, S.; Dalton, A.; Pugno, N. M. Friction and Adhesion of Different Structural Defects of Graphene. ACS Appl. Mater. Interfaces 2018, 10, 44614-44623.

(52) Yan, Z.; Liu, Y.; Ju, L.; Peng, Z.; Lin, J.; Wang, G.; Zhou, H.; Xiang, C.; Samuel, E. L. G.; Kittrell, C.; Artyukhov, V. I.; Wang, F.; Yakobson, B. I.; Tour, J. M. Large Hexagonal Bi- and Trilayer Graphene Single Crystals with Varied Interlayer Rotations. Angew. Chem., Int. Ed. 2014, 53, 1565-1569.

(53) Michail, A.; Parthenios, J.; Anestopoulos, D.; Galiotis, C.; Christian, M.; Ortolani, L.; Morandi, V.; Papagelis, K. Controllable, Eco-Friendly, Synthesis of Highly Crystalline 2D-MoS2 and Clarification of the Role of Growth-Induced Strain. 2D Mater. 2018, 5, No. 035035 .

(54) Shinde, S. M.; Dhakal, K. P.; Chen, X.; Yun, W. S.; Lee, J.; Kim, H.; Ahn, J.-H. Stacking-Controllable Interlayer Coupling and Symmetric Configuration of Multilayered MoS2. NPG Asia Mater. 2018, 10, No. e468.

(55) Lin, X.; Liu, Y.; Wang, K.; Wei, C.; Zhang, W.; Yan, Y.; Li, Y. J.; Yao, J.; Zhao, Y. S. Two-Dimensional Pyramid-like WS2 Layered Structures for Highly Efficient Edge Second-Harmonic Generation. ACS Nano 2018, 12, 689-696.

(56) Gong, Y.; Lin, J.; Wang, X.; Shi, G.; Lei, S.; Lin, Z.; Zou, X.; Ye, G.; Vajtai, R.; Yakobson, B. I.; Terrones, H.; Terrones, M.; Tay, B. K.; Lou, J.; Pantelides, S. T.; Liu, Z.; Zhou, W.; Ajayan, P. M. Vertical and in-Plane Heterostructures from WS2/MoS2 Monolayers. Nat. Mater. 2014, 13, 1135-1142.

(57) Jiang, L. Y.; Huang, Y.; Jiang, H.; Ravichandran, G.; Gao, H.; Hwang, K. C.; Liu, B. A Cohesive Law for Carbon Nanotube/Polymer Interfaces Based on the van der Waals Force. J. Mech. Phys. Solids 2006, 54, 2436-2452.

(58) Girifalco, L. A.; Hodak, M.; Lee, R. S. Carbon Nanotubes, Buckyballs, Ropes, and a Universal Graphitic Potential. Phys. Rev. B 2000, 62, 13104-13110.

(59) Plimpton, S. Fast Parallel Algorithms for Short-Range Molecular Dynamics. J. Comput. Phys. 1995, 117, 1-19.

(60) Nose, S . A Unified Formulation of the Constant Temperature Molecular Dynamics Methods. J. Chem. Phys. 1984, 81, 511-519.

(61) Hoover, W. G. Canonical Dynamics: Equilibrium Phase-Space Distributions. Phys. Rev. A 1985, 31, 1695-1697.

(62) Martyna, G. J.; Tobias, D. J.; Klein, M. L. Constant Pressure Molecular Dynamics Algorithms. J. Chem. Phys. 1994, 101, 4177-

4189 .

(63) Sgouros, A. P.; Kalosakas, G.; Galiotis, C.; Papagelis, K. Uniaxial Compression of Suspended Single and Multilayer Graphenes. 2D Mater. 2016, 3, No. 025033. 
(64) Sgouros, A. P.; Kalosakas, G.; Papagelis, K.; Galiotis, C. Compressive Response and Buckling of Graphene Nanoribbons. Sci. Rep. 2018, 8, No. 9593 .

(65) Tersoff, J. Modeling Solid-State Chemistry: Interatomic Potentials for Multicomponent Systems. Phys. Rev. B 1989, $39,5566$.

(66) Brenner, D. W.; Shenderova, O. A.; Harrison, J. A.; Stuart, S. J.; Ni, B.; Sinnott, S. B. A Second-Generation Reactive Empirical Bond Order (REBO) Potential Energy Expression for Hydrocarbons. J. Phys.: Condens. Matter 2002, 14, 783-802.

(67) Koukaras, E. N.; Kalosakas, G.; Galiotis, C.; Papagelis, K. Phonon Properties of Graphene Derived from Molecular Dynamics Simulations. Sci. Rep. 2015, 5, No. 12923.

(68) Thompson, A. P.; Plimpton, S. J.; Mattson, W. General Formulation of Pressure and Stress Tensor for Arbitrary Many-Body Interaction Potentials under Periodic Boundary Conditions. J. Chem. Phys. 2009, 131, 1-6.

(69) Sgouros, A. P.; Vogiatzis, G. G.; Kritikos, G.; Boziki, A.; Nikolakopoulou, A.; Liveris, D.; Theodorou, D. N. Molecular Simulations of Free and Graphite Capped Polyethylene Films: Estimation of the Interfacial Free Energies. Macromolecules 2017, 50, 8827-8844. 


\section{Supporting Information}

\section{Efficient Mechanical Stress Transfer in Multilayer Graphene with a Ladder-like Architecture}

Aristotelis P. Sgouros ${ }^{1}$, Charalampos Androulidakis ${ }^{2}$, Georgia Tsoukleri ${ }^{2}$, George Kalosakas ${ }^{3}$, Nikos Delikoukos ${ }^{2}$, Stefano Signetti ${ }^{4}$, Nicola M. Pugno ${ }^{4,5}$, John Parthenios ${ }^{2}$, Costas Galiotis ${ }^{2,6}$ and Konstantinos Papagelis $2,7, *$

1 School of Chemical Engineering, National Technical University of Athens (NTUA), Athens 15780 (Greece)

2 Institute of Chemical Engineering Sciences, Foundation for Research and Technology-Hellas (FORTH/ICE-HT), Patras 26504 (Greece)

3

Department of Materials Science, University of Patras, Patras 26504 (Greece)

4

Laboratory of Bio-Inspired, Bionic, Nano, Meta Materials \& Mechanics, Department of Civil, Environmental and Mechanical Engineering, University of Trento,

5 via Mesiano 77, I-38123 Trento, Italy

School of Engineering and Materials Science, Queen Mary University of London, Mile End Road E1 4NS, London, United Kingdom

6

Department of Chemical Engineering, University of Patras, Patras 26504 (Greece)

7

School of Physics, Department of Solid State Physics, Aristotle University of Thessaloniki, Thessaloniki 54124 (Greece) 
to whom correspondence should be addressed. E-mail kpapag@physics.auth.gr

\section{Contents}

S1. Brightness profile and Raman mapping of the studied graphene flake ...............................................2

S2. Uniaxial Raman response under tension of monolayer graphene .................................................

S3. Uniaxial Raman response under tension of bilayer graphene ........................................................

S4. Uniaxial Raman response under tension of trilayer graphene ........................................................

S5. Uniaxial Raman response under compression of trilayer graphene ...................................................

S6. Uniaxial Raman response under tension of four-layer graphene ....................................................11

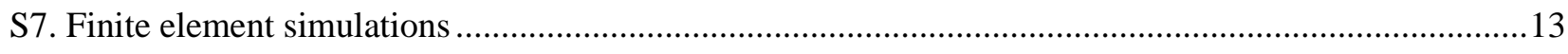

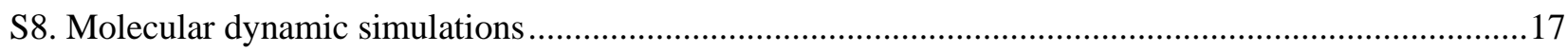

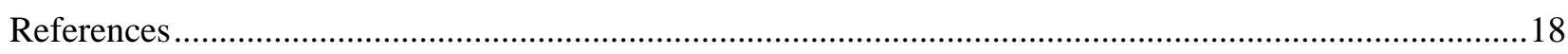

S1. Brightness profile and Raman mapping of the studied graphene flake 


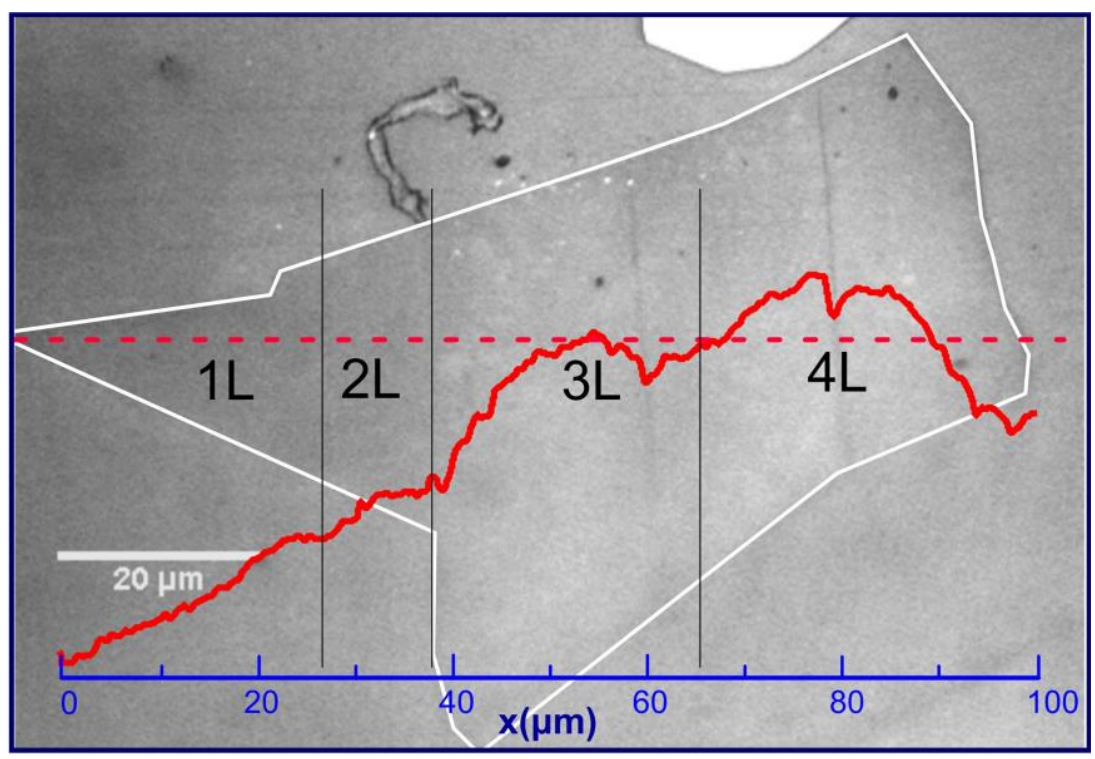

Figure S1. Brightness profile along the dash line designates graphene domains with different thicknesses of the ladder-like graphene flake.

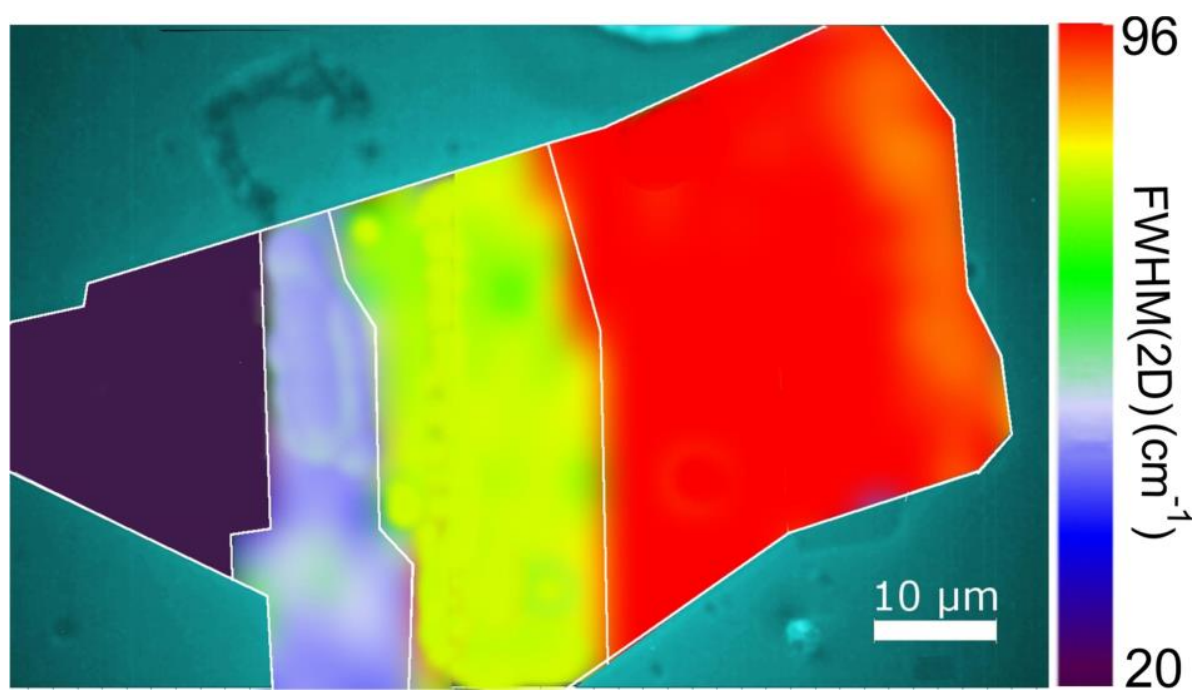

Figure S2. Raman mapping image of the full width at half maximum (FWHM) of the 2D peak for the ladder-like graphene flake. The different color regions correspond to graphene domains with different thicknesses.

The optical contrast difference between the 2D graphene sheet and the substrate was used to identify the number of graphene layers (Figure S1). The brightness profile along the dashed line (Figure S1) generated by the software Image ${ }^{1}$ allowed us to characterise the variation of thickness 
along the flake; the thicker the flake the brighter the image. The elongated flake exhibits four different contrast regions attributed to $1 \mathrm{LG}, 2 \mathrm{LG}, 3 \mathrm{LG}$ and $4 \mathrm{LG}$ as further justified by the results of the Raman mapping (Figure S2).

\section{S2. Uniaxial Raman response under tension of monolayer graphene}

Figure S3(a) shows Raman spectra in the G-peak frequency region for various strain levels up to failure whereas plots of the fitted frequency positions as a function of strain are presented in Figure S3(b). As expected, upon increasing tensile strain the frequency position of $G$ mode shows a distinct redshift. The initial G-peak frequency value at zero applied strain appears at $1583.5 \mathrm{~cm}^{-1}$, indicating that the embedded flake is under a quite small $(\square 0.1 \%)$ residual compressive strain. Embedded graphene into polymers are bound to carry residual compressive strain, due to the fabrication process (mechanical cleavage), the morphology of the underlying substrate and the surrounding film shrinkage due to curing. As it is evident from Figure S3(b), for applied strain higher than $0.2 \%$ the axial stress on graphene lifts the symmetry degeneracy of the phonon, splitting the in-plane optical phonon into two distinct $\mathrm{G}^{-}$and $\mathrm{G}^{+}$components polarized parallel and perpendicular to direction the strain axis, respectively. The extracted strain sensitivities for the $\mathrm{G}^{-}$ and $\mathrm{G}^{+}$with shift rates $-30.1 \mathrm{~cm}^{-1} / \%$ and $-9.4 \mathrm{~cm}^{-1} / \%$, respectively, are comparable to those earlier reported in the literature indicating ideal stress transfer from the matrix to monolayer graphene.

In Figure S3(c) and S3(d) the 2D Raman spectra and the extracted mode frequencies as a function of strain up to $1.46 \%$ are presented for the $1 \mathrm{LG}$. At applied strain of $\sim 0.8 \%$ onset of splitting for the $2 \mathrm{D}$ peak is observed. The shift rate of the higher (lower) frequency component $2 \mathrm{D}^{+}$ $\left(2 \mathrm{D}^{-}\right)$is $-21.5 \mathrm{~cm}^{-1} / \%\left(-62.2 \mathrm{~cm}^{-1} / \%\right)$. 

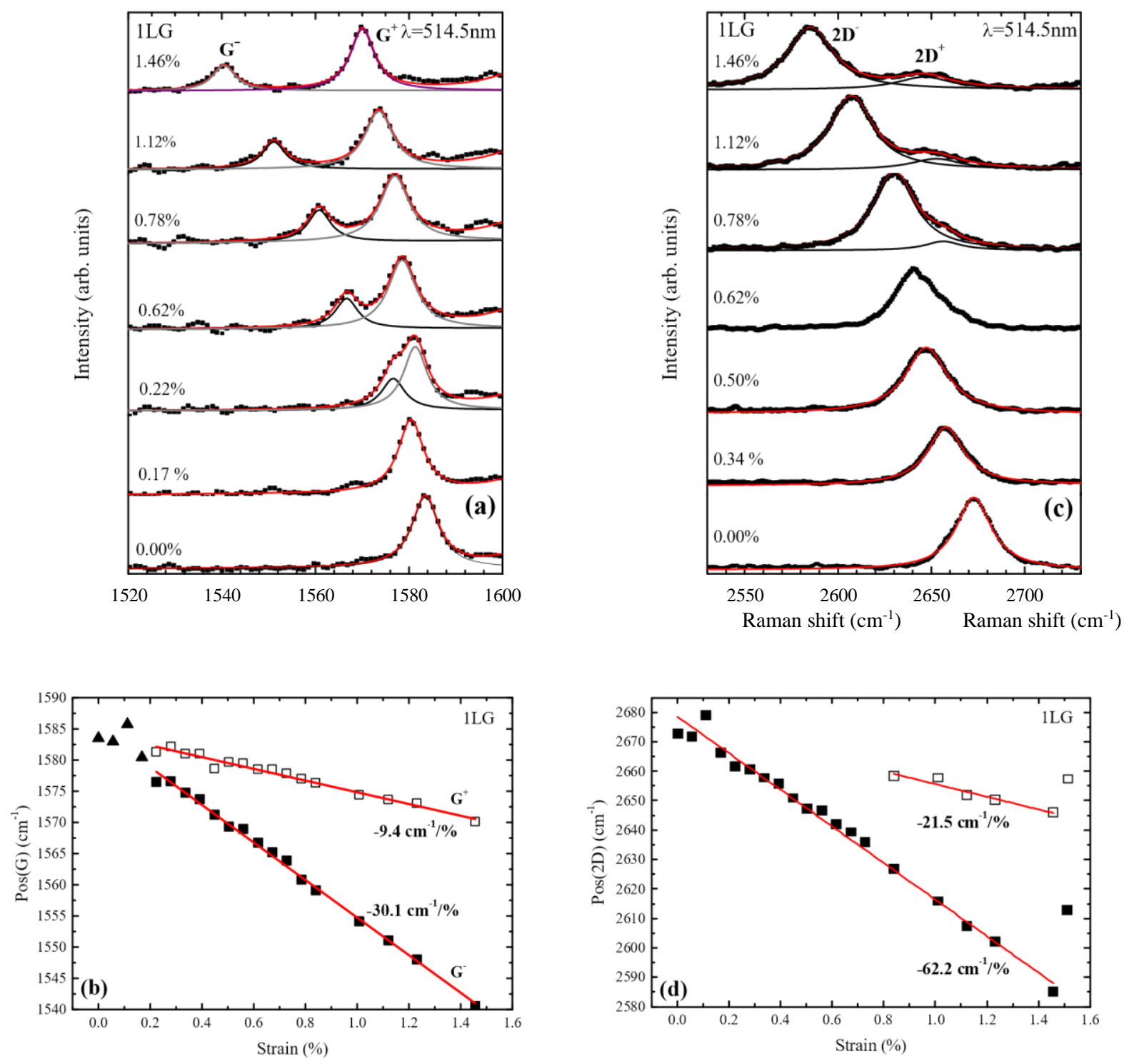

Figure S3. (a) Raman spectra in the $\mathrm{G}$ band region of the monolayer part of the ladder-like sample at room temperature and elevated uniaxial tensile strain. (b) Uniaxial stain dependence of the Raman $\mathrm{G}$ peak using the $514.5 \mathrm{~nm}(2.41 \mathrm{eV})$ excitation of the monolayer region of the ladder-like sample. (c) 2D band Raman spectra under uniaxial tensile strain of the monolayer part of the ladder-like sample. (d) Evolution of the 2D band with strain using the $514.5 \mathrm{~nm}(2.41$ $\mathrm{eV}$ ) excitation of the monolayer region of the ladder-like sample. The phonon frequencies were obtained by fitting Lorentzian functions to the experimental data, after background subtraction. The polarization of the incident beam was parallel to the applied strain axis.

\section{S3. Uniaxial Raman response under tension of bilayer graphene}



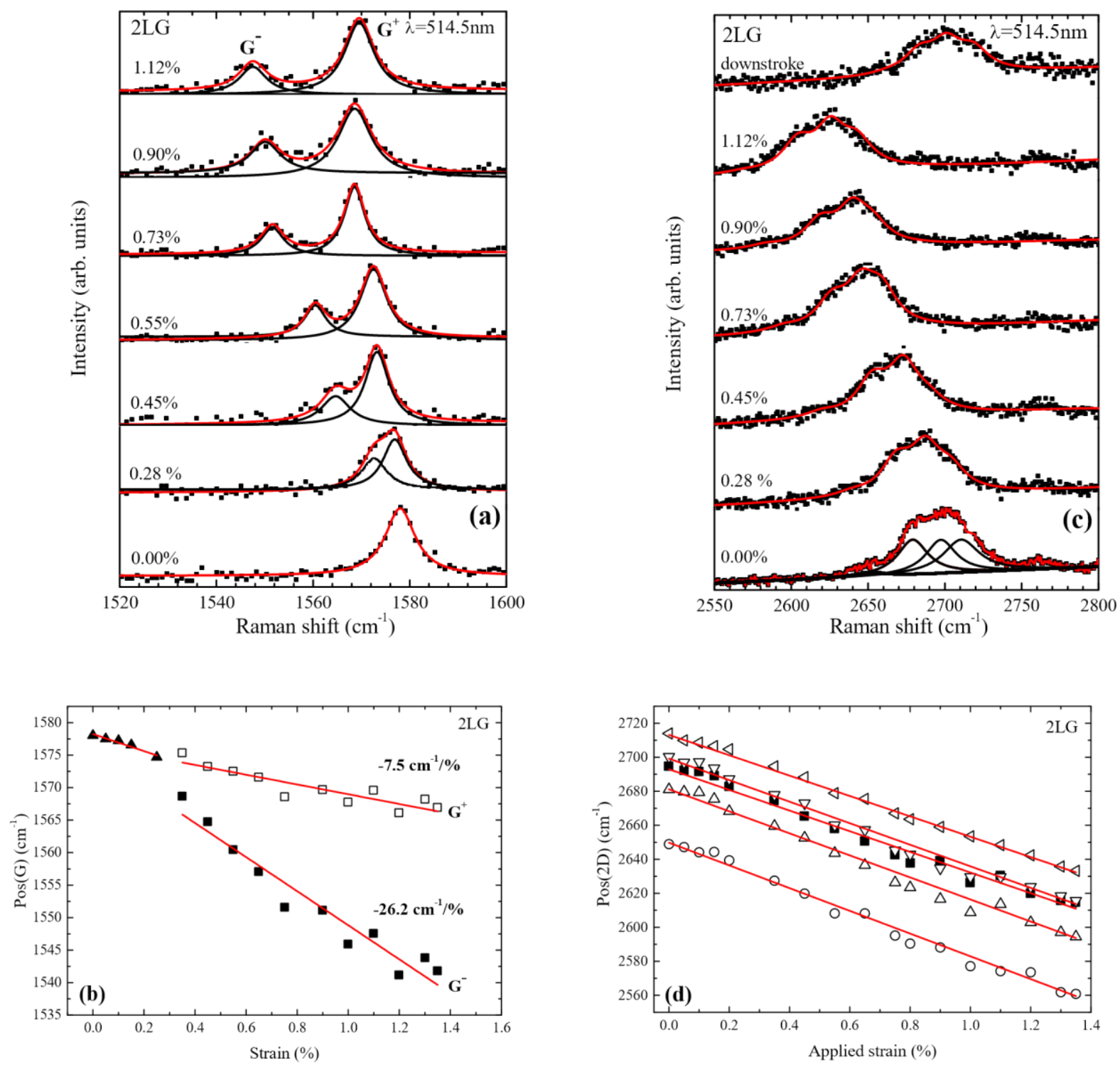

Figure S4. (a) Room temperature G band Raman spectra of the bilayer part of the ladder-like sample, excited at $514.5 \mathrm{~nm}(2.41 \mathrm{eV})$, as a function of uniaxial tensile strain. (b) G-band Raman frequencies at various strain levels of the bilayer part of the ladder-like sample at room temperature. The phonon frequencies were obtained by fitting Lorentzian functions to the experimental data, after background subtraction. (c) 2D Raman spectra of the bilayer part of the ladder-like sample at room temperature at various strain levels, recorded upon strain increase and after strain release (top spectrum). (d) Uniaxial strain dependence of the four Lorentzian components (open symbols) that constitute the Raman 2D peak for the bilayer part of the ladderlike strain, using the $514.5 \mathrm{~nm}(2.41$ $\mathrm{eV}$ ) excitation. The solid symbols correspond to fitting of the $2 \mathrm{D}$ peak by a single Lorentzian function. The polarization of the incident beam is parallel to the applied strain axis.

Bilayer graphene (2LG) contains four atoms per unit cell and possesses $\mathrm{D}_{3 \mathrm{~d}}$ symmetry. From group theory and the compatibility relations, the high frequency optical zone-center mode of 1LG evolves into a Raman active phonon and an infrared-active phonon in the case of $2 \mathrm{LG}^{2}$. Therefore, a single 
doubly degenerate peak can be observed in the Raman spectrum. Figures S4(a) and S4(b) show Raman spectra recorded at elevated strain levels along with the corresponding shift of the $\mathrm{G}^{+}$and $\mathrm{G}^{-}$components. It is clear that at strain of $\sim 0.3 \%$ onset of splitting for the $\mathrm{G}$ band is observed. The 2LG exhibit strain rates of -26.2 and $-7.5 \mathrm{~cm}^{-1} / \%$ for the $\mathrm{G}^{-}$and $\mathrm{G}^{+}$, respectively, up to $1.35 \%$ of strain. These values agree well with previous studies (see Table 1 in the main text).

The 2D band of $2 \mathrm{LG}$ can be deconvoluted into four distinct peaks assigned to four distinct double resonance processes ${ }^{3}$. In Figures S4(c) and S4(d) the 2D Raman spectra and the extracted mode frequencies as a function of strain up to $1.35 \%$ are presented for the $2 \mathrm{LG}$, respectively. All the four 2D components exhibit linear behaviour with strain. The strain sensitivity of the fitted components is ranging between -60.9 and $-66.8 \mathrm{~cm}^{-1} / \%$. Besides by fitting the $2 \mathrm{D}$ profile with a single Lorentzian peak (solid squares in Figure S4(d)) the extracted shift rate is $-60.7 \mathrm{~cm}^{-1} / \%$.

\section{S4. Uniaxial Raman response under tension of trilayer graphene}

3LG exhibits two main stacking order configurations, namely the Bernal (or ABA) and the rhombohedral (or ABC) stacking. The ABA stacking (semimetal) is energetically more stable, and the atoms of the upper layer are located exactly above the atoms of the first layer. On the other hand, the $\mathrm{ABC}$ stacking (semiconductor) is stable for up to $800^{\circ} \mathrm{C},{ }^{4}$ and one of the two upper lattices lies above the centres of hexagons of the bottom layer. The stacking order of 3LG can be easily identified through Raman spectroscopy; in the ABA stacking order the line shape of 2D is more symmetric, while for $\mathrm{ABC}$ stacking the shape is more asymmetric with a sharp peak and an enhanced shoulder. ${ }^{4}$

Figure 2(a) in the main text shows for the embedded trilayer the evolution of the Raman spectra in the G-peak regime under tension, using the $514.5 \mathrm{~nm}$ excitation. The initial G-peak frequency value at zero applied strain appeared at $\sim 1580 \mathrm{~cm}^{-1}$, indicating the absence of residual tensile strain field onto the $3 \mathrm{LG}$. According to group theory ${ }^{2}$, the ABA stacking of trilayer exhibits the $\mathrm{D}_{3 \mathrm{~h}}$ point group and the decomposition for the high frequency optical mode gives $3 \mathrm{E}_{\mathrm{g}} \square 2 \mathrm{E} \square+\mathrm{E} \square \square$. The $E$ is Raman active mode while $E^{\prime \prime}$ is both infrared and Raman active. Due to the poor interlayer 
coupling, however, the splitting is weak ${ }^{2}$. Compared to $1 \mathrm{LG}$ the G-peak in 3LG remains symmetric and only a broadening of the FWHM is detected, namely, from $\sim 7.5 \mathrm{~cm}^{-1}$ for $1 \mathrm{LG}$ to the value of $\sim 10 \mathrm{~cm}^{-1}$ for $3 \mathrm{LG}$.

Figure 2(b) in the main text shows the fitted frequency positions of the G-peak as a function of tensile strain for embedded 3LG. In tension the G peak remains unsplit up to $0.3 \%$ while for higher strain levels $\mathrm{G}^{ \pm}$sub-peaks follow almost linear trends up to the maximum applied strain $(1.15 \%)$. In Figure 2(b) the straight lines for strains higher than $0.3 \%$ are least squared fits to the experimental data giving the values of $-8.5 \mathrm{~cm}^{-1} / \%$ and $-28.4 \mathrm{~cm}^{-1} / \%$ for the $\mathrm{G}^{+}$and the $\mathrm{G}^{-}$components, respectively. It should be stressed that the strain induced lifting of the degeneracy of the $\mathrm{E}_{2 \mathrm{~g}}$ mode in $3 \mathrm{LG}$ have been recently reported by some of the authors ${ }^{5}$ and its presence is crucial for the fundamental distinction of the type of strain (biaxial or uniaxial) applied on a specific graphene sample. This behaviour along with the linear dependence of the $\mathrm{G}^{ \pm}$evolution as well as the magnitude of the corresponding strain coefficients indicates an efficient strain transfer across the graphene-polymer interface. These results extend the application of Raman spectroscopy as a nanometrology tool to quantify the mechanical strain field applied on different types of graphene samples and fabricated graphene-based devices. Moreover, immediately after the applied deflection of $1.15 \%$ the G-peak position has returned to $1580 \mathrm{~cm}^{-1}$. The relaxed $\mathrm{G}$ peak is slightly asymmetric and its FWHM is $\sim 12.5 \mathrm{~cm}^{-1}$ indicating broadening due to local inhomogeneities of the strain field after the full recovery of the strain.

The evolution of the 2D spectra for the $3 \mathrm{LG}$ is shown in Figure $\mathrm{S} 4(\mathrm{c})$. At zero strain the 2D peak profile is symmetric and the examined region exhibits as mentioned above a Bernal ABA stacking configuration. Even though group theory predicts fifteen allowed Raman components to contribute in the 2D peak signal many of these processes turn out to be close to each other and experimentally only six Lorentzians are adequate to fit the 2D spectral feature by the used excitation wavelength ${ }^{6}$. The shift of the six individual components of the 2D peak with tensile strain is shown in Figure S4(d). All the 2D components exhibit linear behaviour with strain. The strain sensitivity of the fitted components ranging between -52.1 and $-60.2 \mathrm{~cm}^{-1} / \%$. The central frequency of the $2 \mathrm{D}$ feature can be determined by the relation $\sum \operatorname{Pos}(2 \mathrm{D}) A$

$\operatorname{Pos}(2 \mathrm{D}) \quad=$

, with $A$ being the frequency integrated intensity ratio 


\section{$\sum A$}

of the i-th peak located at $\operatorname{Pos}(2 \mathrm{D})$, and the sum is extended over all the six sub-peaks used for the deconvolution of the 2D peak. The extracted shift rate of the central frequency is $-57.7 \mathrm{~cm}^{-}$ 1 1\% (solid squares in Figure 2(d)). Similar slopes for 3LG but at much lower strain levels ( $\square 0.4 \%$ ) were found by Gong et al. ${ }^{7}$. The obtained strain sensitivity values for the $\mathrm{G}$ and 2D peaks are similar to the corresponding ones for the $1 \mathrm{LG}$ and $2 \mathrm{LG}$ and higher compared with previously reported values $^{5,7,8}$ for $3 \mathrm{LG}$ under uniaxial tension. We note, that in our previous work, ${ }^{5}$ we have observed a reduced shift rate of the $2 \mathrm{D}$ peak $\left(-43 \mathrm{~cm}^{-1} / \%\right)$ of an embedded $3 \mathrm{LG}$ under uniaxial tension, and a decrease in the shift rate of 3LG compared to thinner graphenes samples under bi-axial tensile deformations. In any case the maximum applied strain $(1.15 \%)$ on $3 \mathrm{LG}$ is the highest achievable in the literature.

The tri-layer part of the flake was stretched up to tensile strain level of $\sim 1.15 \%$. An abrupt upshift of the G (Figures 2(a) and 2(b)) and 2D (Figures 2(c) and 2(d)) peaks occurs at higher strain which indicates failure of the graphene/polymer interface. Loss of Bernal stacking is observed at strain of $\sim 0.73 \%$. Returning the applied deflection back to zero, the shape of $2 \mathrm{D}$ peak does not regain its initial shape, maintaining an unstructured relatively broad peak, implying that the Bernal stacking structural configuration is not fully recovered.

\section{S5. Uniaxial Raman response under compression of trilayer graphene}

In Figure S5(a) an additional ladder-like sample embedded into SU8-PMMA matrix is depicted consisting of a 1L-3L and a multilayer (ML) part. The flake subjected to compressive deformation up to $-0.4 \%$ (positive values correspond to tensile and negative ones to compressive strain). The evolution of the 2D Raman band of the 3LG as a function of compressive strain is shown in Figure S5(b). Each 2D peak is deconvoluted in six components as seen in the $0 \%$ strain level 2D peak. In Figure S5(c) the expected 2D mode frequency hardening upon compressing strain is observed with a strain rate of $59.5 \mathrm{~cm}^{-1} / \%$ up to $-0.15 \%$, where $\operatorname{Pos}(2 \mathrm{D})$ reaches a maximum. For strains larger 
than $-0.15 \%$ (critical buckling strain) the $2 \mathrm{D}$ band softens and the flake cannot not sustain further compression due to structural buckling. 3, 5, 9, 10
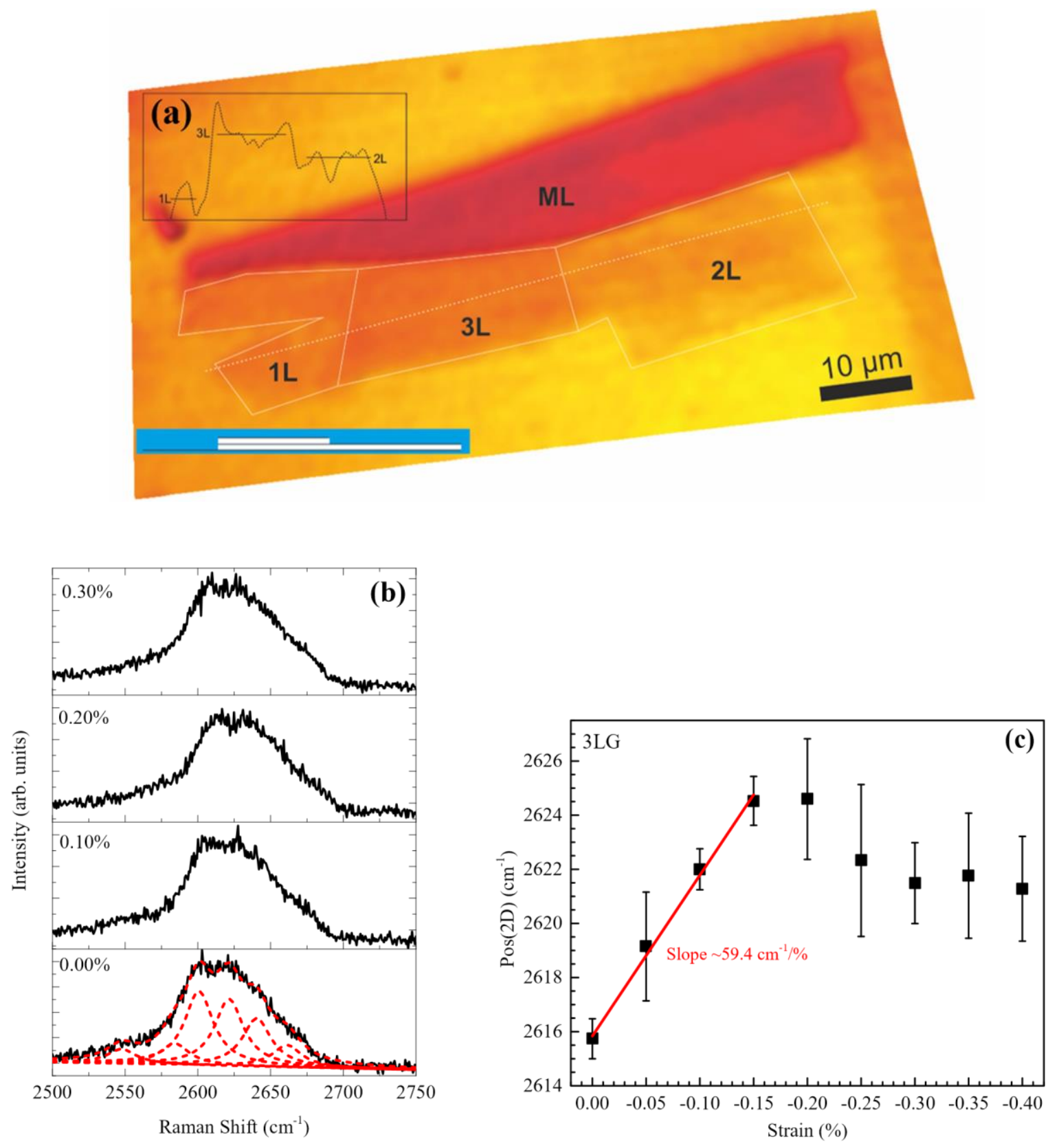

Figure S5. (a) A false color optical micrograph of the embedded graphene ladder-like flake consisting of $1 \mathrm{LG}, 2 \mathrm{LG}, 3 \mathrm{LG}$ and MLG. The inset graph displays the intensity values along the dashed profile line. The relative intensity values are used for the approximate identification of the variation of thickness over the flake. The inset sketch depicts the cross section of the flake along the dashed profile line. (b) 2D band Raman spectra of the trilayer part of the ladder-like sample excited at $514.5 \mathrm{~nm}(2.41 \mathrm{eV})$ under uniaxial compressive strain. The deconvolution of the $2 \mathrm{D}$ band into six distinct peaks is depicted. (c) Pos(2D) versus the applied strain. The error bars of the data points correspond to the standard deviation of five spectra taken from different samples. 


\section{S6. Uniaxial Raman response under tension of four-layer graphene}

The evolution of the $\mathrm{G}$ and 2D peaks of the $4 \mathrm{LG}$ are presented in Figure S6(a) and S6(b), respectively. The maximum achievable strain was $0.75 \%$. The onset of $\mathrm{G}$ splitting begins at about $0.4 \%$, a level slightly higher than the trilayer part of the sample. The strain sensitivities of the $\operatorname{Pos}\left(\mathrm{G}^{-}\right)$and $\operatorname{Pos}\left(\mathrm{G}^{+}\right)$are $-26.7 \mathrm{~cm}^{-1} / \%$ and $-8.1 \mathrm{~cm}^{-1} / \%$, respectively. The $2 \mathrm{D}$ peak, fitted by a single Lorentzian, exhibits a frequency shift of $-53.4 \mathrm{~cm}^{-1} / \%$. The obtained values are high and similar to the rest parts of the flake, indicating that the flake has been mechanically loaded efficiently. After $0.75 \%$ strain level an abrupt decrease of the $G$ and $2 \mathrm{D}$ peak frequencies is observed accompanied by change mainly of the shape of the $G$ spectral profile. This is a clear sign of the failure of interface between graphene and polymer. 

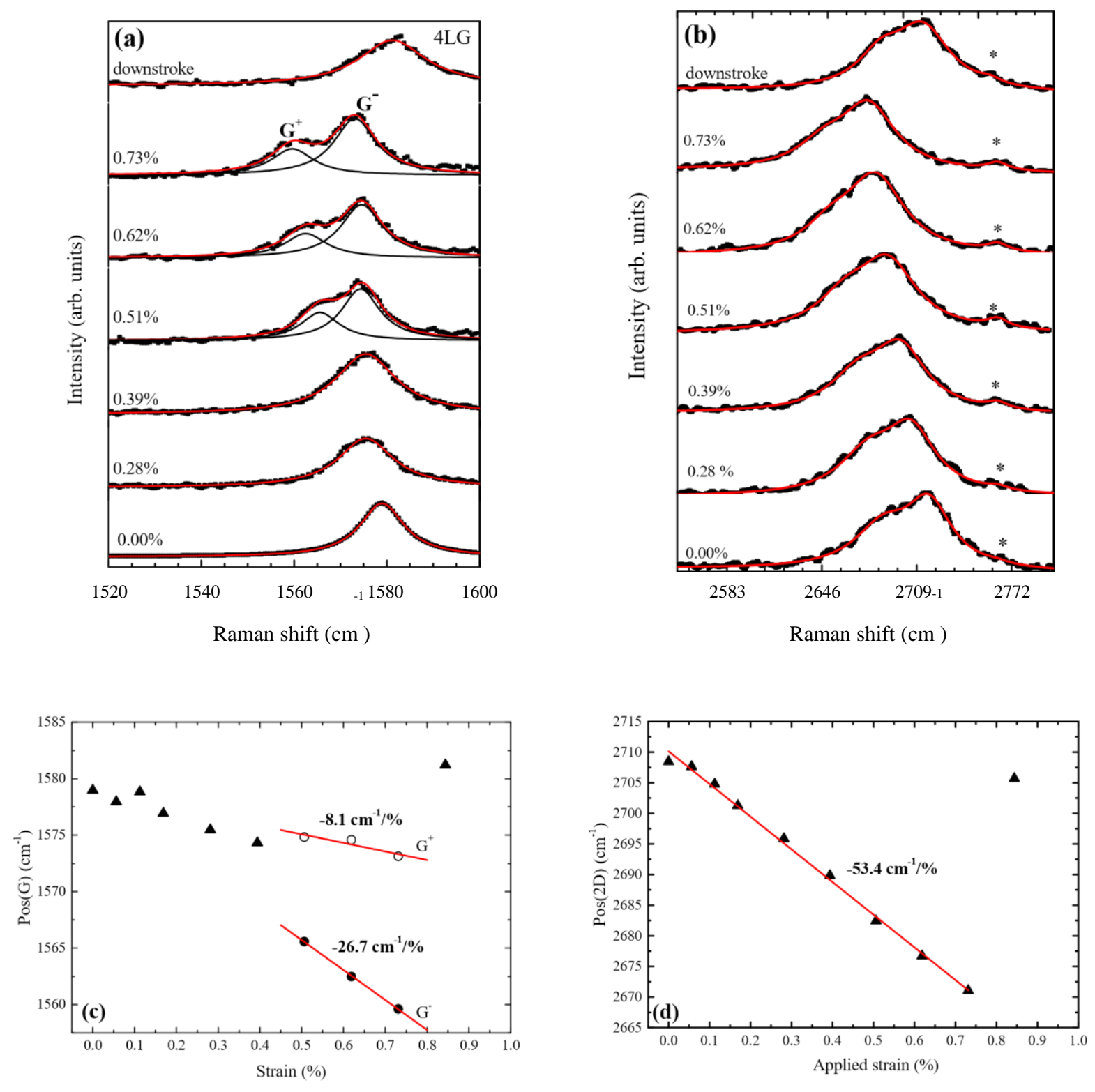

Figure S6. Raman spectra of the four-layer part of the ladder-like sample excited at $514.5 \mathrm{~nm}$ $(2.41 \mathrm{eV})$ at elevated uniaxial tensile strain for (a) the $\mathrm{G}$ and (b) the 2D peaks. The top spectrum in both cases recorded after strain release. The asterisk (*) denotes a Raman peak at $2765 \mathrm{~cm}^{-1}$ originated from the PMMA covering layer. Strain dependence of the frequency positions for (c) the $G$ and $(d)$ the $2 D$ peaks. 
S7. Finite element simulations

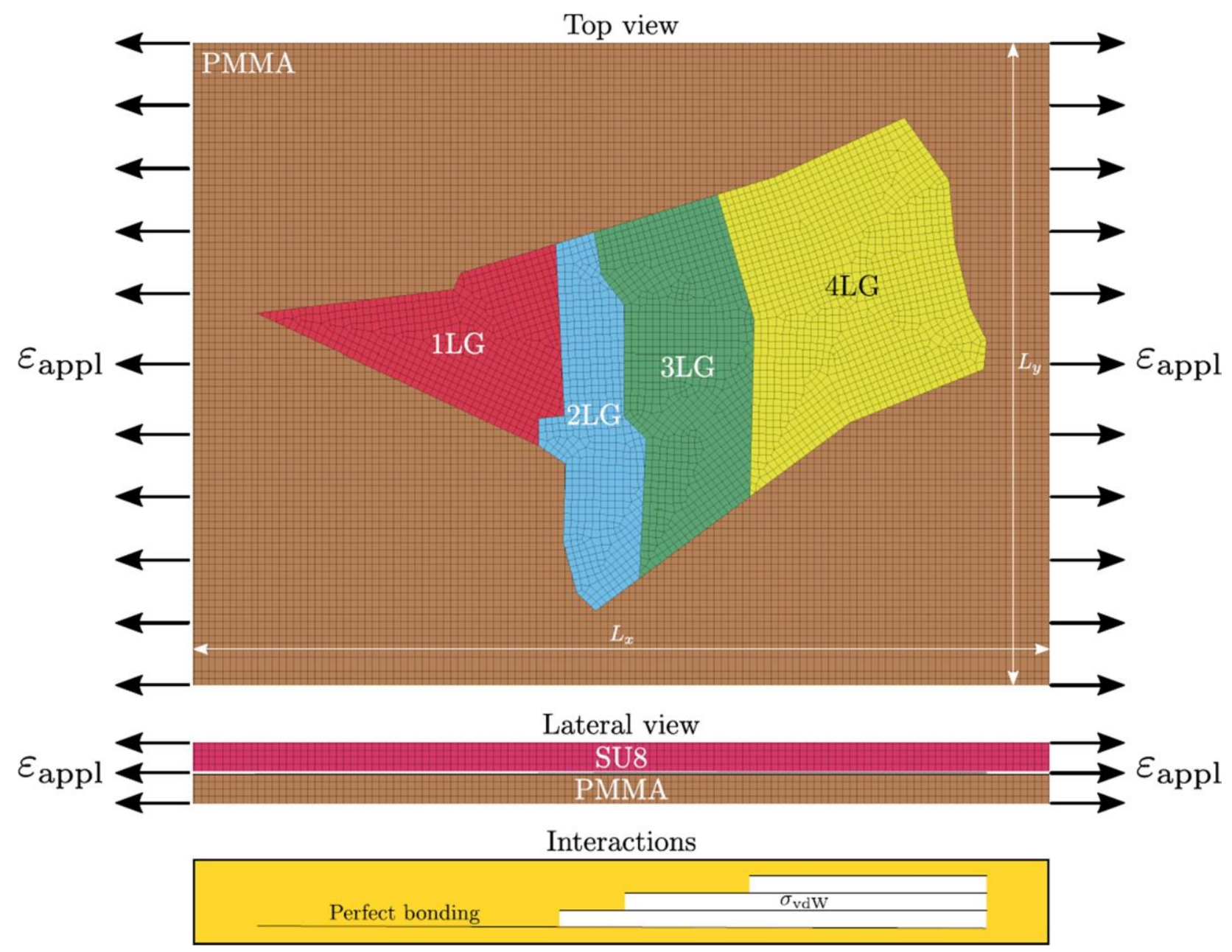

Figure S7. FEM model of the experimentally studied flake of Figure 3a in the main text (geometry from the optical microscopy image of Figure 1(a) in the main text) and schematic representation of the contact interactions between graphene and matrix (perfect bonding) and graphene layers (van der Waals interaction, see Equation (3.a) in the Methods section of the main text). The lateral sizes of the analyzed matrix cell are $L=120 \mu \mathrm{m}$ and $L=90 \mu \mathrm{m}$,

respectively. Interactions and matrix dimension are the same for all tested flake geometries in the work. 
Table S1. Geometrical characteristics of the simulated flakes.

\begin{tabular}{|c|c|c|c|c|}
\hline \multirow{2}{*}{ Flake geometry } & Layer & $\begin{array}{c}\mathrm{L}_{\mathrm{x}} \\
{[\mu \mathrm{m}]}\end{array}$ & $\begin{array}{c}\mathrm{L}_{\mathrm{y}} \\
{[\mu \mathrm{m}]}\end{array}$ & Area $\left[\mu \mathrm{m}^{2}\right]$ \\
\hline \multirow{4}{*}{ Experimental } & 1 & - & - & 3018 \\
\cline { 2 - 5 } & 2 & - & - & 2451 \\
\cline { 2 - 5 } & 3 & - & - & 1966 \\
\cline { 2 - 5 } & 4 & - & - & 1185 \\
\hline \multirow{5}{*}{ Rectangular uniform } & 1 & 74 & 41 & 3034 \\
\cline { 2 - 5 } & 2 & 74 & 41 & 3034 \\
\cline { 2 - 5 } & 3 & 74 & 41 & 3034 \\
\cline { 2 - 5 } & 4 & 74 & 41 & 3034 \\
\hline \multirow{5}{*}{ Rectangular laddered } & 1 & 74 & 41 & 3034 \\
\cline { 2 - 5 } & 2 & 60 & 41 & 2460 \\
\cline { 2 - 5 } & 3 & 48 & 41 & 1968 \\
\cline { 2 - 5 } & 4 & 29 & 41 & 1189 \\
\hline \multirow{5}{*}{ Rectangular double laddered } & 1 & 119 & 41 & 4879 \\
\cline { 2 - 5 } & 2 & 91 & 41 & 3731 \\
\cline { 2 - 5 } & 3 & 67 & 41 & 2747 \\
\cline { 2 - 5 } & 4 & 29 & 41 & 1189 \\
\hline
\end{tabular}




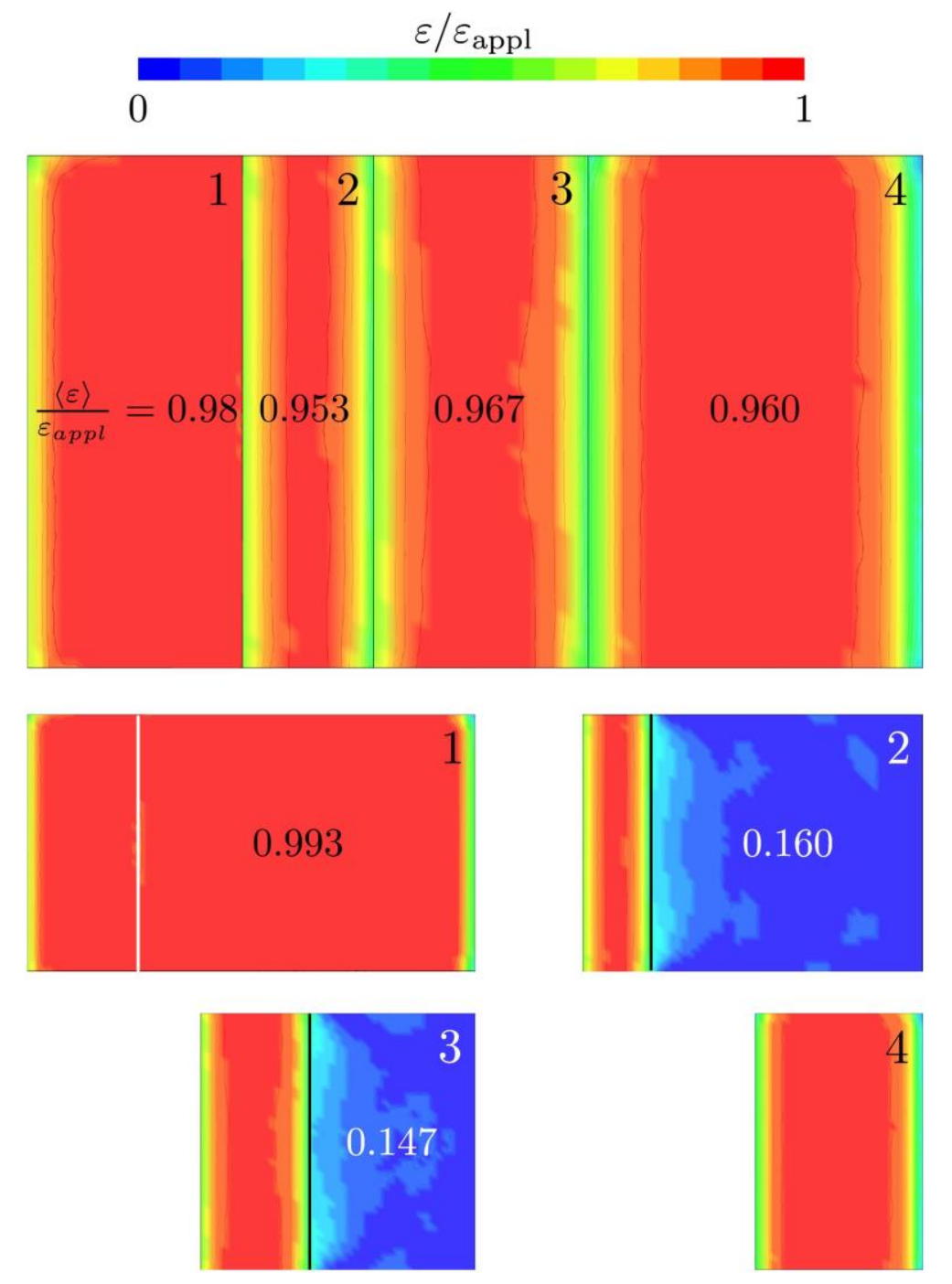

Figure S8. Contour plot of equivalent strain $\varepsilon$ (von Mises) normalized with respect to the applied strain at the boundary of the composite $(\varepsilon)$ for the four layers of the rectangular laddered flake, imitating the experimental configuration of Figure $3 \mathrm{a}$. On each layer, we also indicate the ratio between the average (spatial) strain within the flake $\langle\varepsilon\rangle$ and the applied strain. In the top panel the values refer to the visible areas in contact with the matrix, while in the bottom one the values refer to the regions not visible, which for layers 2 and 3 corresponds to the area not in contact with the matrix. 

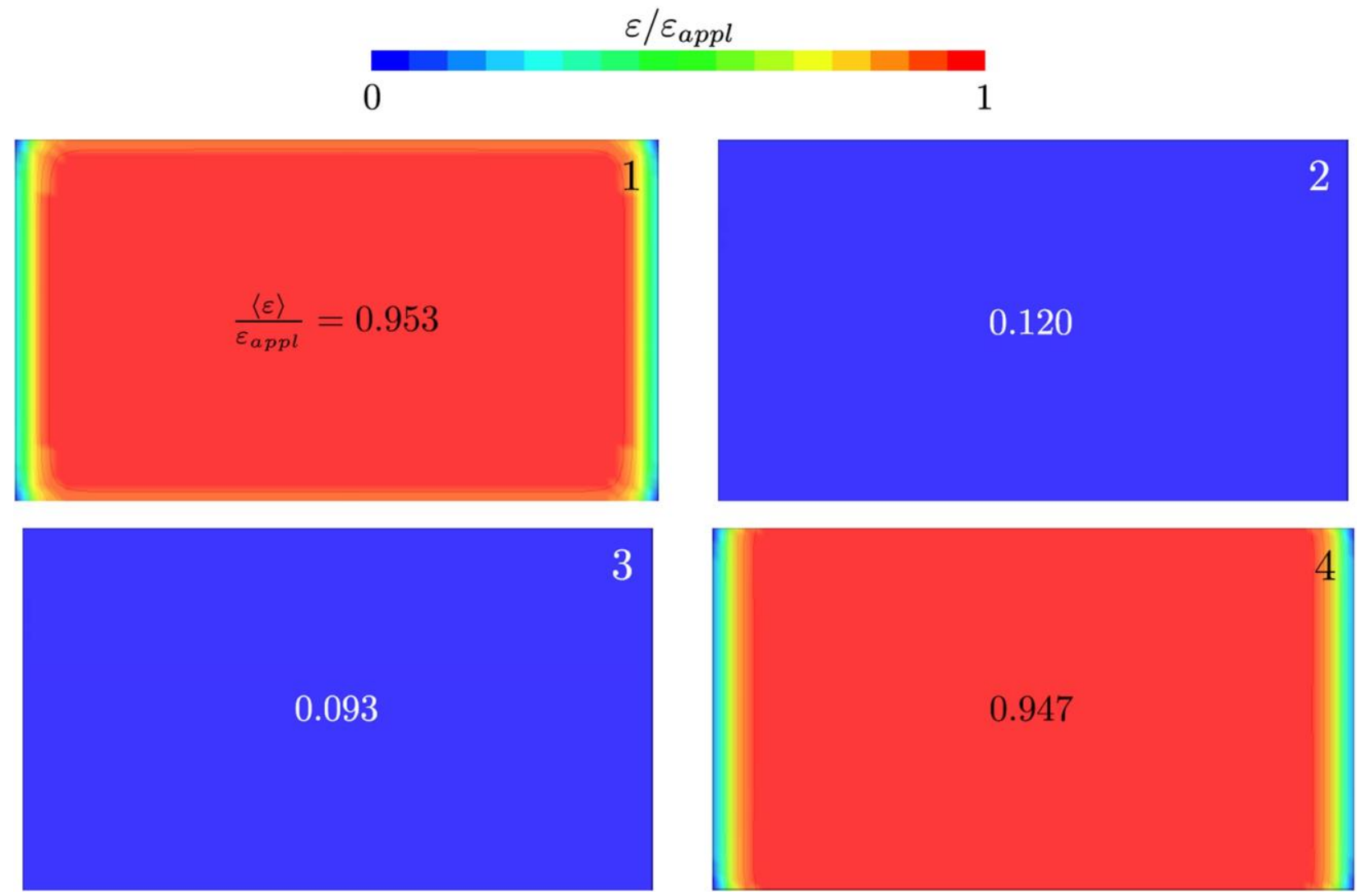

Figure S9. Contour plot of equivalent strain $\varepsilon$ (von Mises) normalized with respect to the applied strain at the boundary of the composite $(\varepsilon)$ for the four layers of the uniform (nonladdered) rectangular flake. On each layer, we also indicate the ratio between the average (spatial) strain within the flake $\langle\varepsilon\rangle$ and the applied strain (see typical configuration in Figure 1c). The differential deformation between the innermost and outermost layers can be clearly observed also by the different longitudinal length (horizontal direction) in the deformed state. 
S8. Molecular dynamic simulations

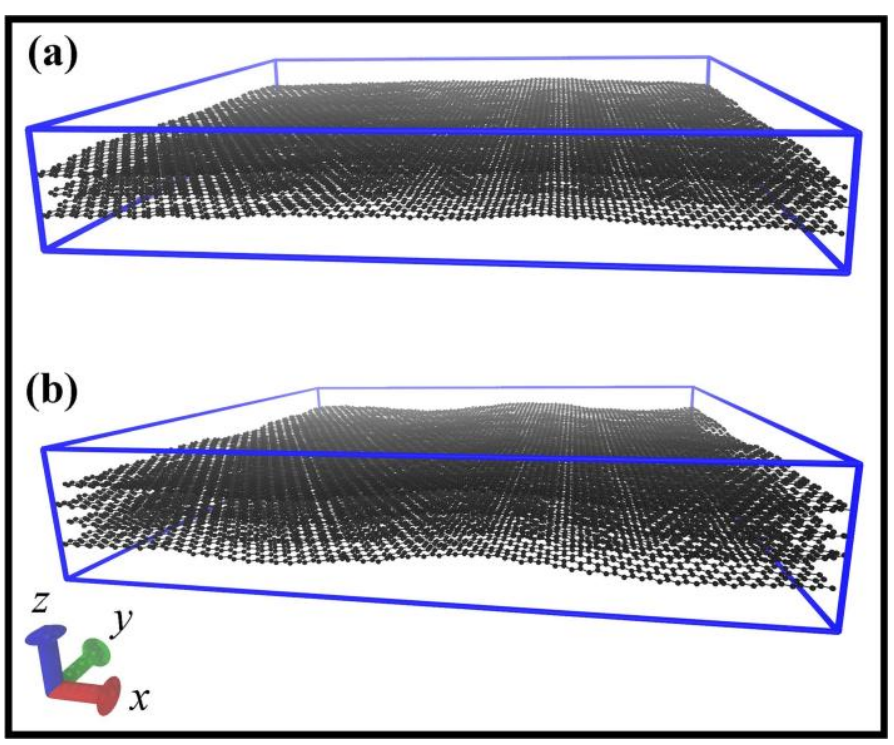

Figure S10. Representations of the atomistic model of (a) the 3LG and (b) the 4LG investigated in the present work. Each layer comprises 5000 carbon atoms and has an area of $\sim 12.3 \times 10.7 \mathrm{~nm}^{2}$.

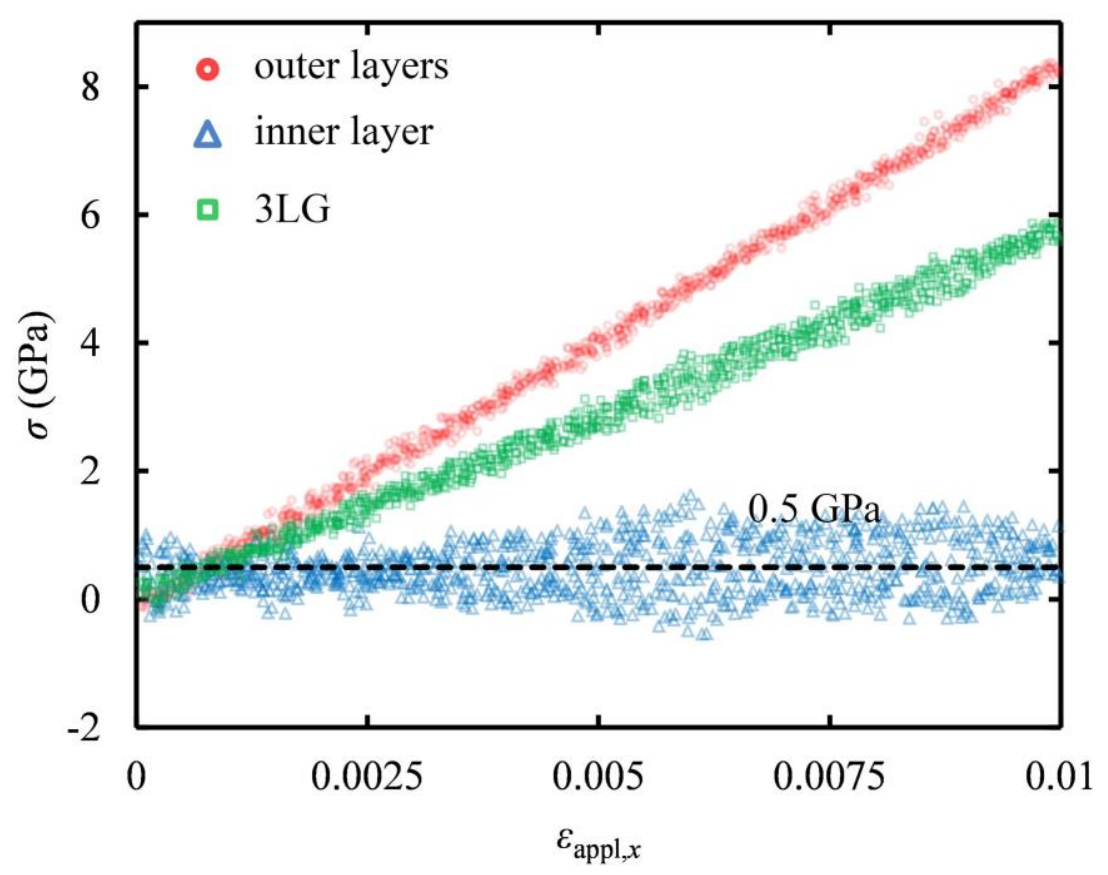


Figure S11. Stress-strain plot extracted from a 3LG with a non-loaded inner layer. The red, blue and green markers depict the stress of the outer, inner and all layers (whole 3LG), respectively. The black dashed line represents the average of the stress in the inner layer.

\section{References}

1. Schneider, C. A.; Rasband, W. S.; Eliceiri, K. W., NIH Image to ImageJ: 25 years of image analysis. Nature Methods 2012, 9 (7), 671-675.

2. Yan, J.-A.; Ruan, W. Y.; Chou, M. Y., Phonon dispersions and vibrational properties of monolayer, bilayer, and trilayer graphene: Density-functional perturbation theory. Physical Review B 2008, 77 (12), 125401.

3. Frank, O.; Bouša, M.; Riaz, I.; Jalil, R.; Novoselov, K. S.; Tsoukleri, G.; Parthenios, J.; Kavan, L.; Papagelis, K.; Galiotis, C., Phonon and Structural Changes in Deformed Bernal Stacked Bilayer Graphene. Nano Letters 2012, 12 (2), 687-693.

4. Lui, C. H.; Li, Z.; Chen, Z.; Klimov, P. V.; Brus, L. E.; Heinz, T. F., Imaging Stacking Order in Few-Layer Graphene. Nano Letters 2011, 11 (1), 164-169.

5. Tsoukleri, G.; Parthenios, J.; Galiotis, C.; Papagelis, K., Embedded Trilayer Graphene Flakes under Tensile and Compressive Loading. 2D Materials 2015, 2 (2).

6. Malard, L. M.; Pimenta, M. A.; Dresselhaus, G.; Dresselhaus, M. S., Raman spectroscopy in graphene. Physics Reports 2009, 473 (5), 51-87.

7. Gong, L.; Young, R. J.; Kinloch, I. A.; Riaz, I.; Jalil, R.; Novoselov, K. S., Optimizing the Reinforcement of Polymer-Based Nanocomposites by Graphene. ACS Nano 2012, 6 (3), 2086-2095.

8. Ni, Z. H.; Yu, T.; Lu, Y. H.; Wang, Y. Y.; Feng, Y. P.; Shen, Z. X., Uniaxial Strain on Graphene: Raman Spectroscopy Study and Band-Gap Opening. Acs Nano 2008, 2 (11), 23012305.

9. Tsoukleri, G.; Parthenios, J.; Papagelis, K.; Jalil, R.; Ferrari, A. C.; Geim, A. K.; Novoselov, K. S.; Galiotis, C., Subjecting a Graphene Monolayer to Tension and Compression. Small 2009, 5 (21), 2397-2402.

10. Androulidakis, C.; Koukaras, E. N.; Hadjinicolaou, M.; Galiotis, C., Non-Eulerian Behavior of Graphitic Materials under Compression. Carbon 2018, 138, 227-233. 